\title{
ChemSpaX: Exploration of chemical space by automated functionalization of molecular scaffold
}

\author{
Adarsh V. Kalikadien ${ }^{1 \odot}$, Evgeny A. Pidko ${ }^{1 *}$, and Vivek Sinha ${ }^{1 *}$ \\ ${ }^{1}$ Inorganic Systems Engineering, Department of Chemical Engineering, Faculty of \\ Applied Sciences, Delft University of Technology, 2629 HZ, Delft, The \\ Netherlands.
}

May 18, 2021

\begin{abstract}
Local chemical space exploration of an experimentally synthesized material can be done by making slight structural variations of the synthesized material. This generation of many molecular structures with reasonable quality, that resemble an existing (chemical) purposeful material, is needed for high-throughput screening purposes in material design. Large databases of geometry and chemical properties of transition metal complexes are not readily available, although these complexes are widely used in homogeneous catalysis. A Python-based workflow, ChemSpaX, that is aimed at automating local chemical space exploration for any type of molecule, is introduced. The overall computational workflow of ChemSpaX is explained in more detail. ChemSpaX uses 3D information, to place functional groups on an input structure. For example, the input structure can be a catalyst for which one wants to use high-throughput screening to investigate if the catalytic activity can be improved. The newly placed substituents are optimized using a computationally cheap force-field optimization method. After placement of new substituents, higher level optimizations using $\times$ TB or DFT instead of force-field optimization are also possible in the current workflow. In representative applications of ChemSpaX, it is shown that the structures generated by ChemSpaX have a reasonable quality for usage in high-throughput screening applications. Representative applications of ChemSpaX are shown by investigating various adducts on functionalized $\mathrm{Mn}$-based pincer complexes, hydrogenation of Ru-based pincer complexes, functionalization of cobalt porphyrin complexes and functionalization of a bipyridyl functionalized cobalt-porphyrin trapped in a M2L4 type cage complex. Descriptors such as the Gibbs free energy of reaction and HOMO-LUMO gap, that can be used in data-driven design and discovery of catalysts, were selected and studied in more detail for the selected use cases. The relatively fast GFN2-xTB method was used to calculate these descriptors and a comparison was done against DFT calculated descriptors. ChemSpaX is open-source and aims to bolster the efforts of the scientific community towards data-driven material discovery.
\end{abstract}

Keywords: catalysis; data-driven material design; density functional tight-binding theory; chemical space exploration; open source;

\section{Introduction}

The discovery of novel molecules is important for many industries. The usage of computational methods for the design or selection of highly selective catalysts has been described as a holy grail of chemistry [1]. Computer-aided drug discovery (CADD) led to the discovery of the HIV 
protease inhibitors ritonavir, indinavir and saquinavir. This discovery proved to be the key in reversing rapid growth in deaths due to AIDS in the US [2,3]. This is one of the many examples that show that CADD has been playing a key role in the discovery of drugs and will continue to do so $[4,5]$. Efforts are being made in the energy and chemicals sector to successfully apply computer-aided methods for discovery of new materials. For example, in the design of materials for lithium-ion batteries, hydrogen production and storage materials, superconductors, photovoltaics and thermoelectric materials $[6,7,8,9]$. A roadmap for materials by computational design is given by Alberi et al., where it is discussed that a common need in material design is the need for high-throughput computational and experimental techniques as a foundation for the materials-by-design paradigm [10].

The chemical space is vast and global exploration of chemical space is difficult [11]. It is experimentally impossible and time-consuming to find the optimal molecule or material from nearly infinite possible combinations. Therefore, machine learning $(\mathrm{ML})$ and other cost-effective computational methods are an attractive solution to the combinatorial problem of material and catalyst design [12]. Von Lilienfeld and coworkers propose that exploration and understanding of chemical space can be done by combining physical theories, data sets of quantum mechanics (QM) and statistical mechanics (SM) properties, and ML methods that incorporate physical and chemical knowledge [13]. These combinations of QM, SM and ML approaches are called QML models. In QML models, modern statistical learning theory is applied to predict electronic and atomistic properties and processess in molecules and materials [13]. However, there are challenges that need to be addressed before a complete workflow for in silico design of chemicals and materials can be achieved.

The first challenge is that computational methods should support scientists in adjusting their hypothesis after synthesis of a material has happened. This is part of the molecular design cycle [14]. To enable this cycle, a systemic approach for the local exploration of the chemical space of the synthesized material is needed to learn more about the chemistry involved. This approach can then be expanded to generate new candidate molecules and adjust the initial hypothesis. By building upon experimental knowledge in a systemic way together with automated computational high-throughput screening (HTS), larger subsets of chemical space can be covered.

The second challenge is that even when accurate simulations are available, the process of molecular design is still limited by the search strategy used to explore chemical space and the representation of a molecule in chemical space [15]. A differentiable continuous space is required to enable the use of gradient-based optimization and make larger jumps in chemical space [15]. An example of molecular representations is shown in literature, where autoencoders are used to map molecule structures onto a continuous latent space. The latent space preserves chemical similarity principle and thus can be used for the generation of analogue structures [16]. Another often used representation is the sorted Coulomb Matrix (CM), this representation was applied successfully in the ML screening of thousands of catalysts [17]. These representations require that the molecular structure that is represented is of reasonable quality, while consumption of computational resources for the generation of the structure is kept to a minimum.

The third challenge is that advances in this field also highly depend on the availability of trustworthy QM data sets. For (small) organic molecules, reliable data sets like the GDB-13 or GDB-11 database exist which are being used to train generative models $[18,19,20]$. In these virtual screening workflows, 3D coordinates are generated from the simplified molecular-input line-entry system (SMILES) strings and these complexes are analyzed further. Large data sets are not readily available in the field of homogeneous catalysis and the alternative approach of using synthetic data generated in silico would become expensive due to the high computational cost of accurate QC methods like DFT [21, 22, 23]. This unavailability stems from the fact that 
transition metal (TM) complexes are regularly used in homogeneous catalysis and it is known that TM-complexes pose an issue for the SMILES, since SMILES strings inherit the imperfections of the valence model [24]. Generating 3D XYZ coordinates from SMILES strings for TM-complexes is an area of active research [25]. In the molsimplify code, a divide-and-conquer technique is used to get the correct 3D geometry of any complex. Force fields for organic components are being used together with a databases of quantum-mechanically derived rules for the metal-organic bonds $[26,27,28,29,30]$. For the exploration of local chemical space for TM complexes, using a SMILES string as starting point is thus something that needs additional research. An alternative to SMILES based chemical space exploration is to directly make use XYZ coordinates of a pre-optimized molecular structure as input. This is the approach that has been taken in this research, which allowed chemical space exploration for any type of geometry.

In this manuscript ChemSpaX, a Python-based workflow that can be used for automated molecular design workflows, is presented. ChemSpaX aims to make the exploration of local chemical space of both organic and inorganic complexes as easy as possible. The exploration is done by automated placement of substituents on a given molecular scaffold while maintaining the quality of the initial scaffold. This process is supposed to simulate the workflow used by experimental chemists in which a molecular structure is tested and explored by placing various functional groups. Several molecular scaffolds are already present in literature and can be used for an automated exploration of local chemical space. If a particular complex is known for its high catalytic activity, the 3D coordinates of this complex can be used as a starting point for exploration in the neighbourhood of its chemical space. With ChemSpaX it is possible to automate this exploration of the local chemical space. The user has full control of the placement of substituents groups and can thus guide the exploration of the local chemical space based on chemical intuition. A general overview of the approach used in ChemSpaX is given in Figure 1.

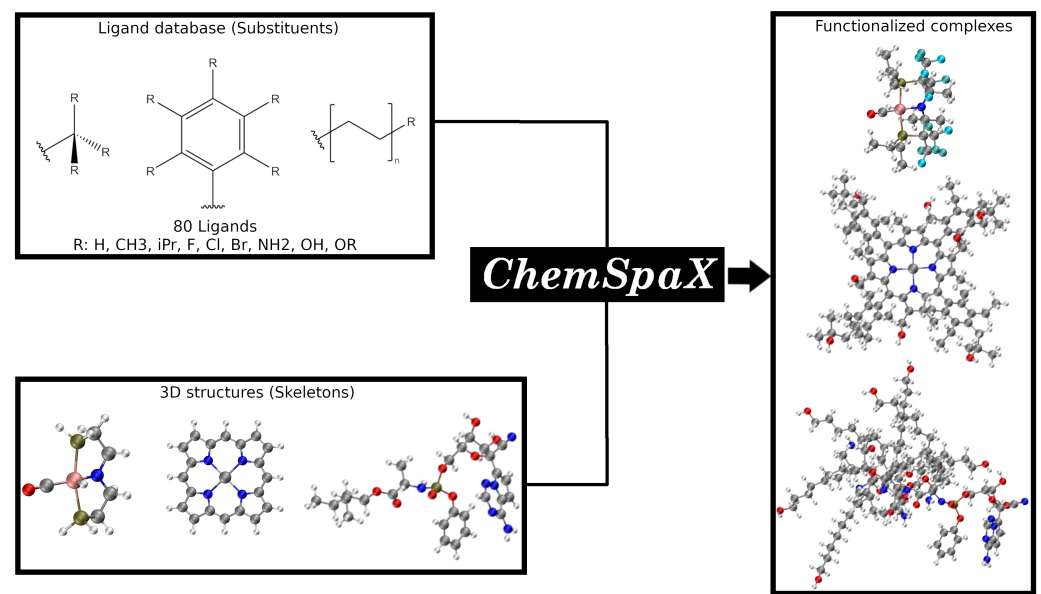

Figure 1: A general overview of the approach used in ChemSpaX. Using a library of pre-defined ligands and a pre-optimized molecular scaffold, the local chemical space of this input geometry can be explored. Color code used for elements: gray $=\mathrm{C}$, white $=\mathrm{H}$, red $=\mathrm{O}$, pink $=\mathrm{Ru}$, dark-blue $=\mathrm{N}$ and turquoise $=\mathrm{F}$. 
The key features of ChemSpaX are presented in the next section. Subsequently, representative applications of ChemSpaX are presented. First, the functionalization of Ru-based complexes with a PNP (PNP $\left.=\left(\mathrm{P}\left(\mathrm{H}_{2}\right) \mathrm{CH}_{2} \mathrm{CH}_{2} \mathrm{~N}\right)_{2}\right)$ backbone (RuPNP) involved in a (de)hydrogenation reaction is studied $[31,32,33,34,35,36]$. This hydrogenated metal-dihydride complex is an important intermediate in catalyzed (de)hydrogenation reactions [37, 38, 39, 40, 41]. These reactions are widely applied in the pharmaceutical, fine chemicals, and energy storage/conversion industries [42, 43, 44, 45, 46, 47]. Analyzing the properties of these RuPNP intermediates by exploration of the chemical space can thus be a valuable asset for multiple applications. For RuPNP, a comparison of the quality of geometries generated by ChemSpaX and calculated electronic or thermochemical properties with higher-level DFT and GFN2-xTB methods is presented.

Secondly, the functionalization of Mn-pincer complexes as potential (de)hydrogenation catalysts is studied. This study is an extension of previous work by our research group [37]. With this application the chemical space of a more climate-friendly alternative to RuPNP is explored. Manganese is known to be a cheap, abundant and biocompatible alternative to precious-metal catalysts [48]. Again, the quality of geometries generated by ChemSpaX is assessed. Furthermore, we also present a thermochemical analysis of formation of various catalytically relevant intermediates and adducts.

Thirdly, the generation of a database of $\sim 1100$ functionalized Cobalt Porphyrin (referred to as 'Co porphyrin' in the rest of this manuscript) complexes is shown. Co porphyrins exist as stable metalloradicals and have been used to catalyze carbene and nitrene transfer reactions $[49,50,51$, 52]. Automated generation of this database shows the possibility of systemic exploration of local chemical space. The generated database is used to investigate quality of geometries generated by ChemSpaX and the propagation of errors upon creation of larger geometries. Additionally, a comparison of electronic properties calculated by GFN2-xTB and DFT is made.

Lastly, the functionalization of a bipyridyl functionalized Cobalt-porphyrin trapped in a M2L4 type cage complex (referred to as 'M2L4 cage' in the rest of this manuscript) is presented. This cage complex confines the Co porphyrin catalyst, which can lead to changed catalyst properties $[53,54,52]$. This case shows how ChemSpaX can be used to automatically functionalize structures that are more difficult to functionalize. The Root-mean-square deviation of atomic positions (RMSD) of various optimization methods is compared for the M2L4 cage.

\section{Computational methods}

\subsection{Open Babel}

Conversions between MDL Molfile and $X Y Z$ format were done using Open Babel [55, 56]. For structures generated by ChemSpaX the Generalized Amber Force Field (GAFF) followed by the Universal Force Field (UFF) optimization method as implemented in Openbabel was used $[57,58]$. This order of optimization gave a reasonable geometry based on pre-defined molecular connections.

\subsection{Semiempirical tight-binding}

Grimme lab's $x$ TB package (version 6.3.3) was used for extended DFTB calculations [59]. The GFN2-xTB method and GFN-FF methods were used for geometry optimization [60, 61, 62, 63]. The RuPNP geometries were optimized using an extended density functional tight-binding approach, by applying the GFN2-xTB method with verytight criteria. Hessian calculations were also performed for these geometries to verify the absence of imaginary frequencies and that 
each geometry corresponds to a local minimum on its respective potential energy surface (PES). The Mn-pincer complexes and Co porphyrins were optimized using GFN2-xTB without hessian calculations. The M2L4 cage geometries were optimized using GFN2-xTB and GFN-FF.

The GBSA solvation method as implemented in $\times$ TB was used with THF as solvent for most optimizations, to implicitly account for solvent effects [64, 65]. These GFNn $(n=0,1,2, F F)$ methods are denoted as GFNn-xTB(THF) or GFNn-xTB(GAS) depending on whether GBSA solvation was used.

\subsection{Density Functional Theory}

\subsubsection{Pincer complexes}

Gaussian 16 C.01 was used to perform DFT calculations [66]. The BP86 exchange-correlation functional was used for geometry optimizations together with the def2SVP basis set $[67,68]$. This combination of functional and basis set have shown reliable geometry predictions accompanied with low computational costs [69, 70]. Geometry optimizations were performed in the gas phase. Hessian calculations were performed for these geometries to verify the absence of imaginary frequencies and that each geometry corresponds to a local minimum on its respective potential energy surface (PES). Thermochemical parameters such as the Gibbs free energy were calculated using the gas phase hessian. Single point (SP) DFT calculations were performed on the gas-phase optimized geometries using the SMD solvation (THF) model [71]. SP calculations were performed using BP86 or PBE1PBE (also known as PBE0) functional with the def2TZVP basis set to further refine the obtained (free) energies and other thermochemical/electronic properties [72]. All DFT calculations were performed with Grimme's D3 dispersion corrections [73]. These composite methods, BP86/def2-SVP//XC/def2-TZVP (THF), are denoted as XC(THF) or XC(GAS) depending on the exchange-correlation (XC) functional used and if a SP calculation with solvation was done.

All geometries were pre-optimized with a combination of Openbabel's GAFF and UFF methods or GFN2-xTB before being subjected to full DFT based optimization. In this research no conformational search was conducted. The catalysts are represented as $M-L$ where $M$ represents the metal center and $L$ the ligand. This lead to the formation of $M(X)-L(H)$ species. The thermodynamic stability of the formed $M(X)-L(H)$ species was estimated by computing the Gibbs free energy and total energy change under standard conditions upon addition of the $\mathrm{H}-\mathrm{X}$ moiety.

$$
\begin{array}{r}
\mathrm{H}-\mathrm{X}+\mathrm{M}-\mathrm{L} \longrightarrow \mathrm{M}(\mathrm{X})-\mathrm{L}(\mathrm{H}) \\
\Delta G_{\mathrm{HX}}^{\circ}=G(M(X)-L(H))-G(M-L)-G(\mathrm{H}-\mathrm{X}) \\
\Delta E_{\mathrm{HX}}^{\circ}=E(M(X)-L(H))-E(M-L)-E(\mathrm{H}-\mathrm{X})
\end{array}
$$

\subsubsection{Co porphyrins}

TeraChem v1.94V-2019.08-beta was used to perform GPU-accelerated DFT SP calculations $[74,75,76]$ on selected Co-Porphyrin geometries optimized using the GFN2-xTB method. The PBE1PBE exchange-correlation functional was used with empirical dispersion corrections $[72,77]$ in combination with the LANL2DZ basis set which uses an effective core potential (ECP) [78].

\subsection{Root-mean-square deviation of atomic positions (RMSD)}

The RMSD is used to compare two molecular structures. In this approach, the minimal difference between the position of the same atom on both molecular structures is used. The RMSDs were calculated using a Python program which uses the Kabsch or Quaternion algorithm to align the two molecular structures $[79,80,81]$. If for example the two molecules $\mathbf{p}$ and $\mathbf{q}$ with $\mathrm{n}$ points (atoms) are compared, the RMSD is defined as: 


$$
\begin{aligned}
\operatorname{RMSD}(\mathbf{p}, \mathbf{q}) & =\sqrt{\frac{1}{n} \sum_{i=1}^{n}\left\|p_{i}-q_{i}\right\|^{2}} \\
& =\sqrt{\frac{1}{n} \sum_{i=1}^{n}\left(\left(p_{i x}-q_{i x}\right)^{2}+\left(p_{i y}-q_{i y}\right)^{2}+\left(p_{i z}-q_{i z}\right)^{2}\right)}
\end{aligned}
$$

\section{Code implementation}

ChemSpaX is a python tool that allows the automated functionalization of molecular structures, aimed at easing the creation of an automated workflow for quantum chemistry calculations. An overview of the overall workflow of ChemSpaX as described in this section is shown in Figure 2. The user has to supply: a molecule that needs to be functionalized (skeleton), which sites on the skeleton should be functionalized (functionalization_list) and what substituent should be placed on the supplied site (substituent). Substituents can be chosen from a pre-made database included in ChemSpaX or users can supply new substituents in $X Y Z$ or MDL Molfile format. Based on the user input, the substituent is placed optimally on the skeleton. Information for the correct placement of a substituent is kept in a CSV file, here the central atom of the substituent group and its centroid vector are stored. After a geometrically correct placement, the GAFF and UFF optimization methods from Open Babel are used to selectively optimize the newly placed substituent via a constrained optimization protocol $[55,56]$. In this constrained optimization protocol, the skeleton is frozen. This combination of GAFF and UFF was found by trial-and-error. The choice for this combination is explained in the SI.

It is recommended to use a DFT optimized geometry as input skeleton. When a new functional group is placed on the skeleton, the skeleton itself is fully constrained, hence the FF optimization only influences the newly placed functional group. This choice was made to keep the core of the geometry as close to its DFT optimized input structure as possible while preventing steric hindrance from newly placed substituents cheaply. The resulting geometries can be used for screening purposes or can be further optimized using semi-empirical methods or DFT. 

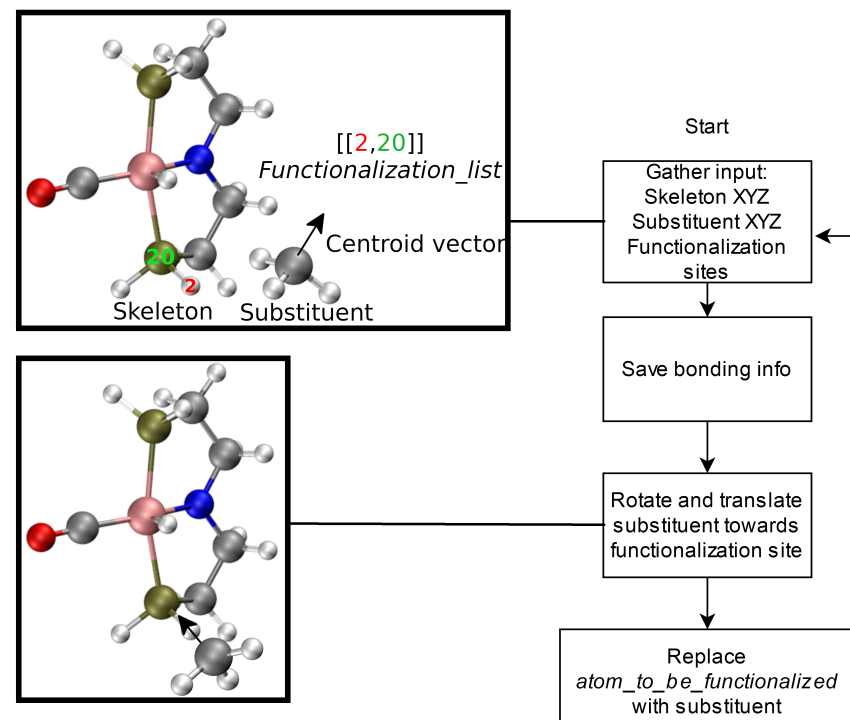

\section{Start}

Gather input: Skeleton XYZ
Substituent XYZ Functionalization

sites

$\downarrow$ with substituent

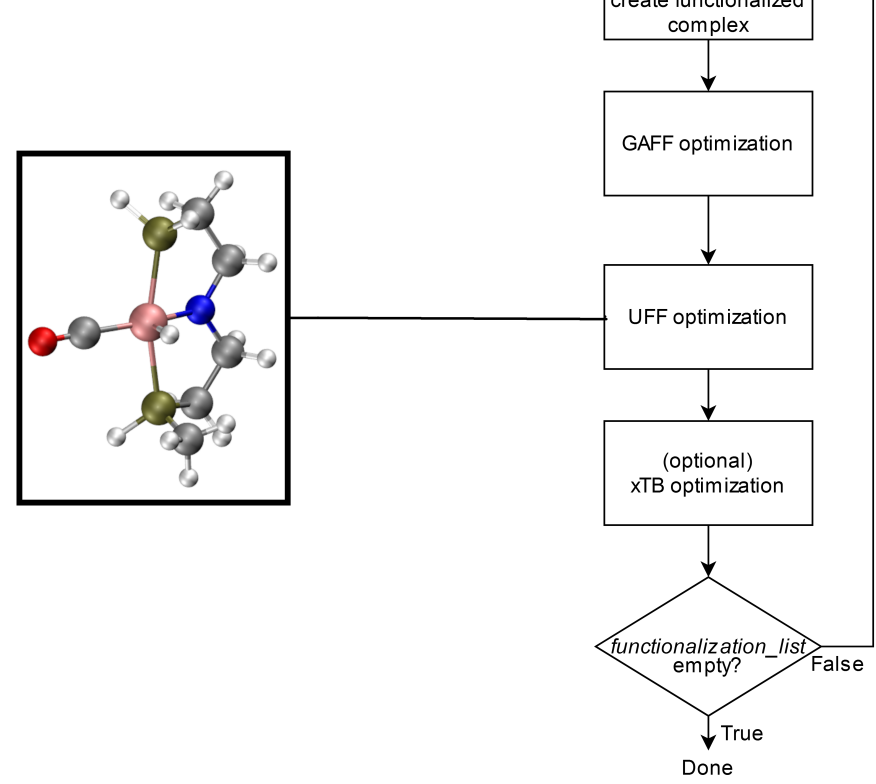

Merge substituent
and skeleton to and skeleton to
create functionalized

Figure 2: Overall workflow of ChemSpaX. 1) The user supplies a skeleton $X Y Z$, functionalization_list and substituent. 2) The $X Y Z$ files are converted to MDL Molfiles to conserve correct bonding info. 3) The central atom of the substituent group and the centroid vector are used to rotate and translate the substituent group towards the functionalization site. 4) atom_to_be_functionalized is replaced by the substituent group. 5) The skeleton and substituent group are merged in one MDL Molfile with correct bonding information from input MDL Molfiles. 6) GAFF optimization is done to prevent steric hindrance. 7) Additionally, UFF optimization is done to prevent GAFF related issues. 8) Optionally, xTB optimization can be used for further optimization of the functionalized skeleton. 9) If there are no functionalizations left to do, the program is done and the functionalized skeleton is saved in MDL Molfile format. Else the functionalized skeleton will be used as input and the process starts again at step 1 . 


\section{Results and discussion}

\subsection{Pincer complexes}

In this section the functionalization of the ligand scaffold of Ru and $\mathrm{Mn}$ based pincer complexes was investigated. For all pincer complexes the activated catalyst $(M-L)$ has a Lewis acid site on the metal and the ligand can act as a Bronsted base. This means that the metal can coordinate with an electron donating species while the ligand can accept a proton [37, 38]. For the (de)hydrogenation reactions, the outer-sphere Noyori-type mechanisms, involving proton transfer to the amido ligand and hydride transfer to the metal center are typically proposed. For the Mn-pincers, potential deactivation/inhibition through the metal-ligand cooperative addition of alcohol/water/base are studied [37]. The properties of the pincer complexes' intermediate with an electron donating species on the metal and a proton on the ligand are thus an important factor to ensure that the desired product is synthesized. The Noyori-type mechanism involved in these reactions together with an example 3D structure of the Mn-pincer are shown in Figure 3.

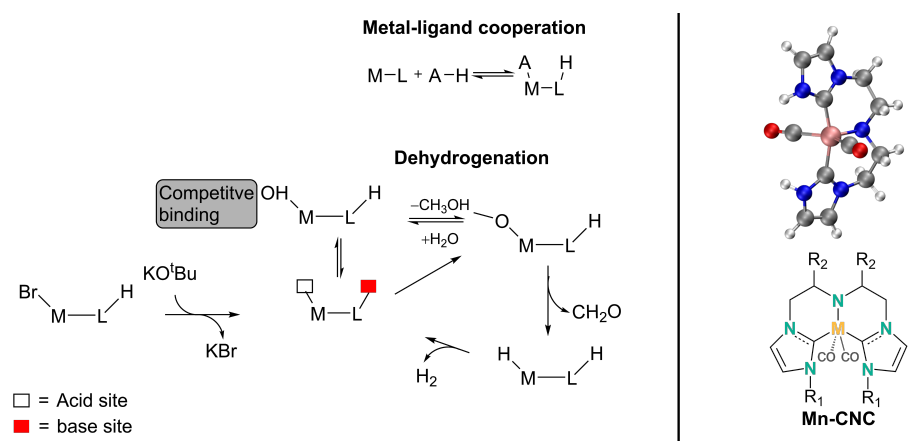

Figure 3: A representative proposed Noyori type cooperative catalytic cycle for dehydrogenation of methanol in aqueous phase is shown (left) together with an example Mn-pincer complex (right). Color code used for elements: gray $=\mathrm{C}$, white $=\mathrm{H}$, red $=\mathrm{O}$, pink $=\mathrm{Mn}$, dark-blue $=$ N.

In section 4.1.1 the functionalization strategy for both pincer complexes is shown. Subsequently, the quality of geometries generated by ChemSpaX is compared to higher level methods in section 4.1.2. An investigation of thermochemical is done in section 4.1.3. A comparison of the HOMO-LUMO gap calculated using XTB versus DFT is shown in the SI.

\subsubsection{Functionalization strategy}

The Ru-based complexes had a PNP backbone coordinated to a Ru(II) center stabilized by Co and $\mathrm{H}$ ligands shown in Figure 4. For these RuPNP complexes, 288 geometries were generated by functionalization of the $M-L$ complex and the $M(H)-L(H)$ variant. 27 geometries were selected for BP86(GAS) optimization. BP86(THF) and PBE1PBE(THF) single-point (SP) calculations were done on the optimized geometries. Generally, the phosphorus sites were functionalized first and the carbon moieties on the PNP ligand backbone were functionalized second. This strategy is shown in Figure 4.

For the Mn-based complexes five representative ligand scaffolds were considered, PNP(bis(3-phosphaneylpropyl)amine)- ${ }^{1}$, SNS-(azanediylbis(ethane-1-thiol))-, CNC-(bis(2-(1H-3 $\lambda^{4}$ -

\footnotetext{
${ }^{1}$ This is not the same PNP ligand as used for the Ru complexes. The PNP ligand used for Mn complexes contains a propyl bridge while the one used for Ru complexes contains an ethyl bridge
} 


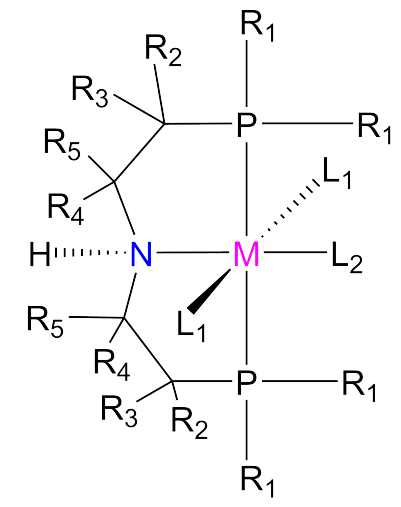

$$
\begin{aligned}
& \mathrm{R}_{\mathrm{i}}=\left\{\mathrm{Me}, \mathrm{Et},{ }^{\mathrm{i}} \mathrm{Pr}, \mathrm{Ph}, \mathrm{CF}_{3}\right\} ; \mathrm{L}_{1}=\mathrm{H} \\
& \mathrm{L}_{2}=\left\{\mathrm{CO}, \mathrm{PMe}_{3}\right\} ; M=\mathrm{Ru}
\end{aligned}
$$

Figure 4: Functionalization strategy for the RuPNP pincer complexes.

imidazol-3-yl)ethyl)amine)-, PNN-(N1-(2-phosphaneylethyl)ethane-1,2-diamine)-, and PCP$\left(\mathrm{N}^{1}, \mathrm{~N}^{3}\right.$-bis(phosphaneyl)benzene-1,3-diamine)- backbones coordinated to a $\mathrm{Mn}(\mathrm{I})$ center stabilized by two $\mathrm{CO}$ ligands. 1225 geometries were generated using ChemSpax and optimized using GFN2-xTB(THF). Functionalizations were done on the $M-L$ and $M(X)-L(H)$ complex. From these 1225 geometries, 545 geometries containing the PCP, PNN and CNC ligand backbones were selected for DFT optimization using BP86(GAS). BP86(THF) (SP) calculations were done on the optimized geometries. Functionalizations were performed symmetrically, all four R1 sites were kept the same and both R2 sites (only 1 in case of PCP backbone) were functionalized with the same group. However, R1 and R2 were not constrained to be the same. The various backbones and the functionalization strategy are shown in Figure 5.

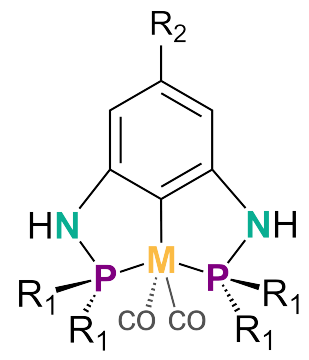

PCP<smiles>[R]C1CP([R7])(=O)N2C([R])CN([R17])N12</smiles>

PNN

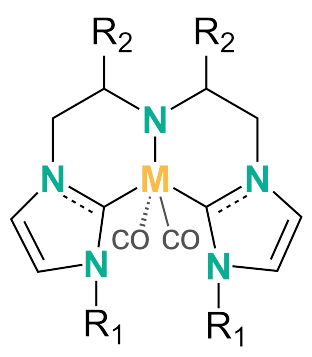

$\mathrm{CNC}$

$$
\begin{gathered}
M=M n \\
R_{i}=\left\{C F_{3}, H, P h, i P r, c y, t B u\right\}
\end{gathered}
$$

Figure 5: Functionalization strategy for Mn-pincers with various donor (R1) and backbone (R2) groups. 


\subsubsection{Quality assessment of generated geometries}

For data-driven chemical applications it is desirable to generate a large number accurate 3D geometries at a rapid pace. TTherefore, a computationally efficient method which can produce molecular geometries close to full DFT optimized geometries is desirable [17, 82, 83]. Methods like FF, xTB and genetic algorithms (GA) are attractive solutions for in situ structure generation. For this work all input skeletons were optimized at the DFT level of theory. ChemSpaX uses FF optimizations on newly placed substituent groups in each iteration, hence these geometries are referred to as 'FF geometries' in this manuscript. In this work the quality of the ChemSpaX generated FF geometries and GFN2-xTB optimized geometries was assessed by comparison with DFT optimized structures. These DFT optimized geometries were used as a 'standard' for comparison.

We applied two methods to compare the quality of geometries. First, the electronic energy of reaction was used for comparison. In this comparison, for a given reaction the ChemSpaX generated FF geometries of reactants and product are taken. Each FF geometry undergoes two calculations: 1) a SP calculations where the energy of the FF geometry is evaluated at DFT level of theory and the electronic energy change of the reaction $\Delta \mathrm{E}_{\mathrm{FF} / / \mathrm{DFT}-\mathrm{SP}}$ is calculated 2) a full DFT based optimization is carried resulting in a new geometry and energy at the DFT level of theory. The corresponding electronic energy change of the reaction $\Delta \mathrm{E}_{\mathrm{DFT}}$ is calculated. The difference between the reaction energies is computed using both $\Delta \mathrm{E}_{\mathrm{FF} / / \mathrm{DFT}-\mathrm{SP}}$ and $\Delta \mathrm{E}_{\mathrm{DFT}}$ to get $\Delta \Delta \mathrm{E}_{\mathrm{FF}}$ which is a metric for the quality of $\mathrm{FF}$ geometry for the reaction being investigated. A similar approach was used in a previous work from our group to assess GFN2-xTB optimized geometries [84].

$$
\begin{aligned}
\Delta \Delta E_{G F N 2-x T B} & =\Delta E_{D F T}-\Delta E_{G F N 2-x T B / / D F T-S P} \\
\Delta \Delta E_{F F} & =\Delta E_{D F T}-\Delta E_{F F / / D F T-S P}
\end{aligned}
$$

For RuPNP, $\Delta \Delta \mathrm{E}_{\mathrm{FF}}$ and $\Delta \Delta \mathrm{E}_{\mathrm{GFN} 2-\mathrm{xTB}}$ were compared. The mean $(\mu)$ of the $\Delta \Delta \mathrm{E}_{\mathrm{FF}}$ was found to be $7.20 \mathrm{kcal} \mathrm{mol}^{-1}$ with a standard deviation $\left(\sigma^{2}\right)$ of $4.57 \mathrm{kcal} \mathrm{mol}^{-1}$. For $\Delta \Delta \mathrm{E}_{\mathrm{GFN} 2-\mathrm{xTB}} \mu=4.77 \mathrm{kcal} \mathrm{mol}^{-1}$ with $\sigma^{2}=2.57 \mathrm{kcal} \mathrm{mol}^{-1}$ was found. This indicates an overall good agreement between the GFN2-xTB optimized structures and the structures generated by ChemSpaX. For the Mn-pincer complexes only the $\Delta \Delta \mathrm{E}_{\mathrm{FF}}$ was calculated, which resulted in $\mu=27.2 \mathrm{kcal} \mathrm{mol}^{-1}$ and a very high $\sigma^{2}$ of $26.1 \mathrm{kcal} \mathrm{mol}^{-1}$. The FF geometries were compared against BP86(GAS) optimized geometries The large difference in between FF and DFT based reaction energies for Mn pincers indicate that unlike for the Ru-PNP complexes the geometries of $\mathrm{Mn}$-pincers generated by ChemSpaX are relatively less accurate.

The second methods used to assess the quality of geometries was RMSD between geomteries produced by two different methods. For the RuPNP complexes, the Cartesian heavy-atom (all elements except $\mathrm{H}$ ) root-mean-square deviation (hRMSD) was calculated [59]. With this method the average distance between two geometries (in $\AA$ ) is calculated. Again, DFT optimized structures were used as the standard for comparison. Both FF $\left(\mu=0.67 \AA ; \sigma^{2}=0.30 \AA\right)$ and GFN2-xTB $\left(\mu=0.41 \AA ; \sigma^{2}=0.34 \AA\right)$ structures had a similar average hRMSD when compared to DFT structures. As observed with the electronic energy, the structures generated by ChemSpaX are in good agreement with GFN2-xTB optimized structures for RuPNP complexes. Generally, it was observed that the hRMSD's were mostly close to $1 \AA$ and never exceeded 2.5 $\AA$, both for FF and GFN2-xTB. Distributions of the hRMSD values are shown in the SI.

A selection of the geometries are visualized using structure overlay plots in Figure 6. The comparisons in these structure overlay plots are done in a similar way as calculating the RMSD, a) the FF optimized structure (silver), generated by ChemSpaX, is compared to a DFT opti- 
mized structure (green) and b) a GFN2-xTB optimized structure (silver) is compared to a DFT optimized structure (green). FF optimization on newly placed substituents only as employed by ChemSpaX can thus generate promising geometries for HTS applications since the geometries are in reasonable agreement with higher-level methods like GFN2-xTB while being relatively less resource consuming.
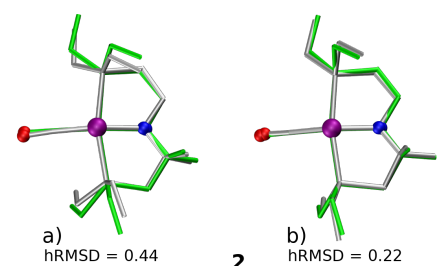

$R_{1}=E t, R_{4}^{2}=R_{5}=M e$

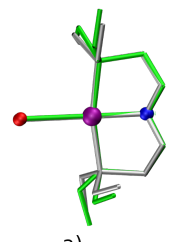

$$
\text { a) }
$$
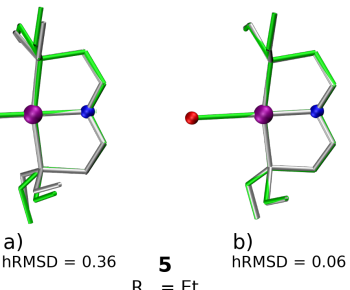

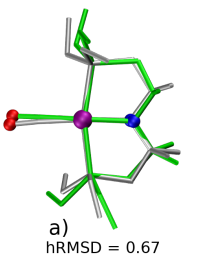

$R_{1}=E t, R_{4}^{3}=R_{5}=M e$
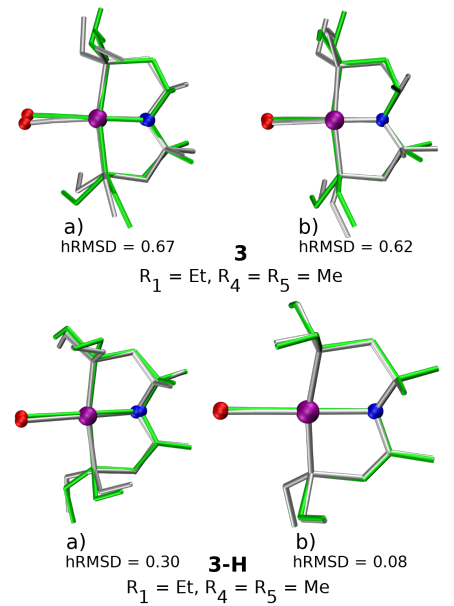
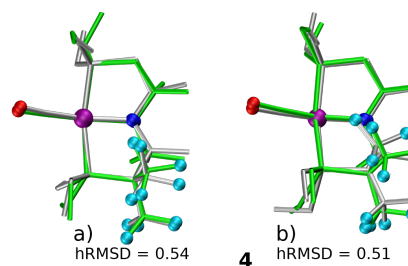

$\mathrm{R} 1=\mathrm{Me}, \mathrm{R}_{4}=\mathrm{R}_{5}=\mathrm{Me}, \mathrm{R}_{2}=\mathrm{R}_{3}=\mathrm{CF}_{3}$

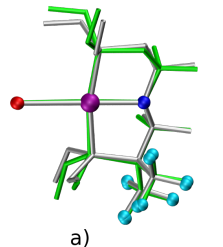

a)

$R_{1}=M e, R_{4}=R_{5}=M e, R_{2}=R_{3}=C F_{3}$

Figure 6: Structure overlay plots of some selected TM complexes. a) shows FF optimized (silver) vs DFT optimized (green) structures and b) shows GFN2-xTB optimized (silver) vs DFT optimized (green) structures. The '-H' indicates that the complex is hydrogenated. Color code used for elements: red $=\mathrm{O}$, purple $=\mathrm{Ru}$, dark-blue $=\mathrm{N}$ and turquoise $=\mathrm{F}$.

A comparison using the hRMSD was done in a similar manner for the Mn-pincer complexes. It was again observed that both FF $\left(\mu=0.70 \AA ; \sigma^{2}=0.45 \AA\right)$ and GFN2-xTB $\left(\mu=0.78 \AA ; \sigma^{2}\right.$ $=0.67 \AA$ ) structures had a similar average hRMSD when compared to DFT structures, albeit with moderately high standard deviations. This is consistent with the previous observation from the RuPNP structures, that the structures generated by ChemSpaX are in good agreement with GFN2-xTB optimized structures. It should be noted here that the GFN2-xTB optimziations were performed in the solvated phase (GBSA(THF)).

The hRMSD of ligands bonded to the metal center was also compared for various backbones. This comparison is shown in Figure 7. As observed in the previous work, most PNN based complexes resulted in hemilabile ligands, this hemilability could arise as an artifact of XTB based geometry optimization or it can be genuinely present in the system [37]. Due to this hemilability, the spread of the PNN backbone hRMSD data was larger compared to the other backbones. For the PNN and CNC backbones it was observed that functionalization with electron donating substituents on the R1 site resulted in a higher hRMSD. For the PCP backbone it was observed that functionalization with $t \mathrm{Bu}$ on the R1 site specifically gave a larger hRMSD. This observation is expected to have the following underlying causes: 1) electron donating groups like $t \mathrm{Bu}$ are bulkier, have more number of atoms and a complex structure which increases the chance of accumulating an error (vide infra) 2) electron donating groups affect the electronic density in the entire complex, this will impact the structure of the skeleton which is currently frozen in FF calculations. When optimized with DFT, the skeleton would relax and this would lead to a higher hRMSD. 

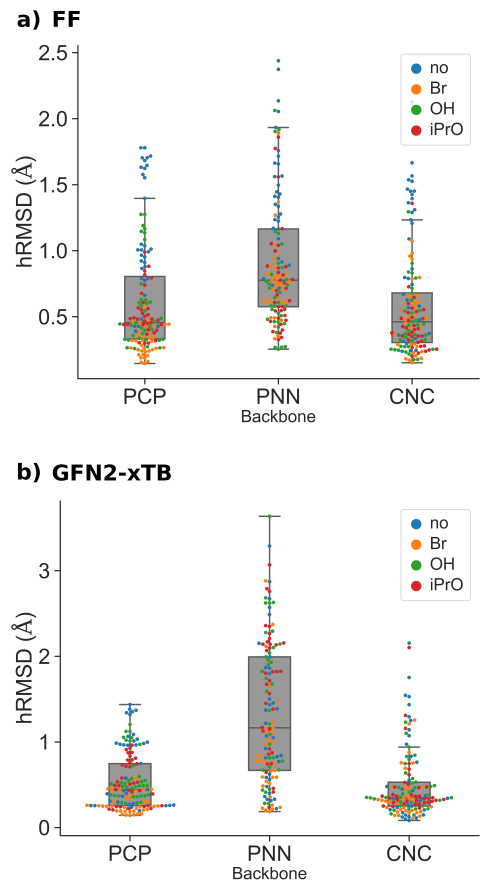

Figure 7: Comparison of hRMSD for the PCP, PNN and CNC ligand backbone of a) ChemSpaX generated FF structures compared to DFT optimized structures, b) GFN2-XTB optimized structures compared to DFT optimized structures. The various adducts bonded to the metal center are color coded, where 'no' means that no adduct is bonded to the metal center.

\subsubsection{Comparison of $\Delta \mathrm{G}$ and $\Delta \mathrm{E}$}

Calculating the hessian is necessary to find the Gibbs free energy of reaction $\Delta \mathrm{G}$. Hessian calculation is also quintessential to ensure accurate and reliable geometry optimizations. However, it would be desirable to omit these computationally expensive calculations when filtering through a large number of molecular structures in high-throughput screening (HTS). In a previous study done by our group, it was observed that $\Delta \mathrm{G}$ correlates well with $\Delta \mathrm{E}$ for the proton transfer reaction [84]. In the present work we found a similar correlation to hold for the Ru-PNP and $\mathrm{Mn}$-pincers (Figure 8. This correlation shows that $\Delta \mathrm{E}$ scales well with $\Delta \mathrm{G}$ and due to the observed minimal loss of accuracy, $\Delta \mathrm{E}$ can be used instead of $\Delta \mathrm{G}$ [84]. Thus, heavy resourceconsuming hessian calculations can be skipped without losing a significant amount of accuracy in HTS applications (RMSE (Ru-PNP) $=1.7 \mathrm{kcal} \mathrm{mol}^{-1}$;RMSE (Mn-pincers) $=1.9 \mathrm{kcal} \mathrm{mol}^{-1}$ ). We would like to emphasize here that for accurate calculations hessian calculations must be performed to ensure that the optimized geometry is a minima on the potential energy surface. 

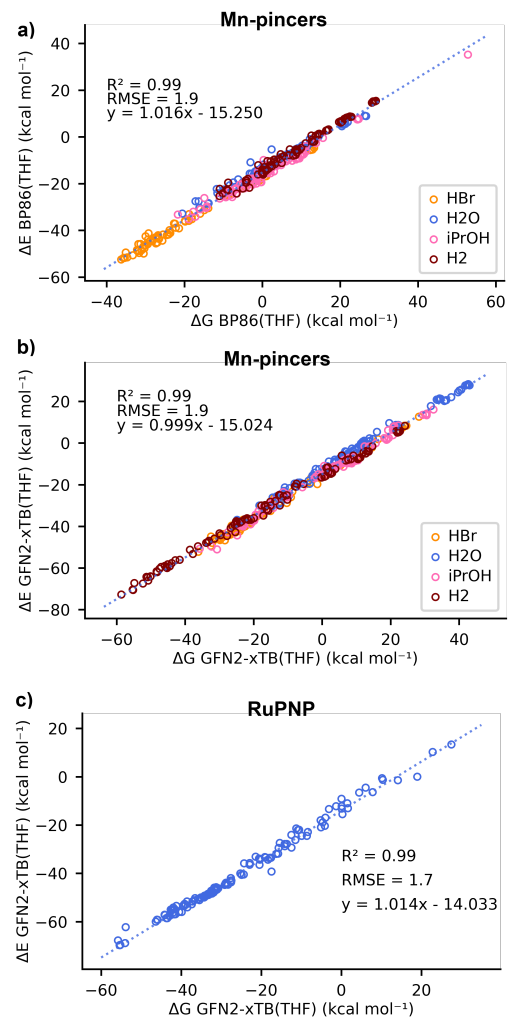

Figure 8: Comparison between the Gibbs free energy of reaction $(\Delta \mathrm{G})$ and the electronic energy of reaction $(\triangle \mathrm{E})$ in a) DFT calculations for Mn-pincers, b) GFN2-xTB calculations for Mnpincers and c) GFN2-xTB calculations for RuPNP [84].

\subsection{Co porphyrin}

Porphyrins are widely researched, for example, for their applications in biocatalysis, organic photovoltaics, molecular wires and many more $[85,86,87,88]$. This wide variety of applications has been enabled by diverse to design and synthesis of structurally diverse porphyrins [85]. Postfunctionalization is a common method to tune the electronic and chemical properties of porphyrins, where functional groups and substituents are introduced after the construction of the porphyrin macrocycles. However, experimental exploration of the chemical space of porphyrins is limited by synthetic and economic feasibility. This is where computer-aided molecular design tools can be helpful.

Apart from the chemical point of view, investigating the functionalization of porphyrins is also of use for further refinement of our workflow. When functionalizing a structure as implemented in ChemSpaX (freezing the skeleton and performing FF optimization only on the newly placed substituents), errors can be introduced. Stretching or compression of the skeleton structure is not taken into account since the skeleton is frozen. Relaxation of the skeleton upon placement of a new substituent group is thus barely taken into account. By investigating a structure that is close to $2 \mathrm{D}$ instead of $3 \mathrm{D}$, like porphyrins, the assessment of the introduced errors and their propagation through the workflow of ChemSpaX is simplified. 
We performed functionalization of Co porphyrins following a serial functionalization strategy ${ }^{2}$ to create a database of $\sim 1100$ complexes.Section 4.2.1 discusses the functionalization strategy. Subsequently, the propagation of errors introduced in geometries generated by ChemSpaX is investigated in section 4.2.2. An investigation of the HOMO-LUMO gap is done in section 4.2.3.

\subsubsection{Functionalization strategy}

Figure 9 shows the functionalization strategy for Co porphyrin, the Co porphyrin skeletons were functionalized with various phenyl groups on the R1 sites to generate 10 skeletons. These skeletons were then used to generate 1120 functionalized Co porphyrin complexes. The functionalization was done serially as described in Code implementation. The sites X1-X5 on the phenyl rings (R1) were functionalized first. Finally, functionalizations were done on R2 and R3 respectively. The resulting complexes are shown for 3 different skeletons in Figure 10, where the

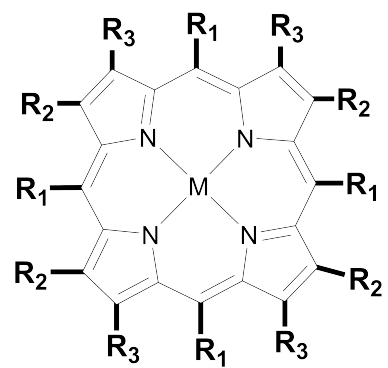<smiles>[Y]c1c([Y4])c([Y4])c([Y])c([Y])c1[Y4]</smiles>

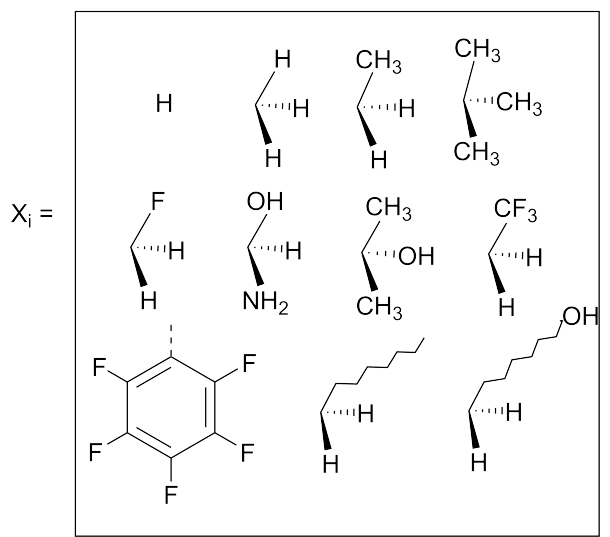

Figure 9: Functionalization strategy for Co porphyrin, phenyl groups were placed on the R1 sites and these newly placed phenyl groups were the first targets for functionalization. With this strategy a database of 1120 Co porphyrin structures was generated.

skeleton, 5th functionalization and 15th functionalization are shown in a column demonstrating the geometric complexity introduced upon functionalization.

\footnotetext{
${ }^{2} \mathrm{~A}$ serial functionalization strategy means the functional group were placed one after another leading to more complex functionalized structures.
} 


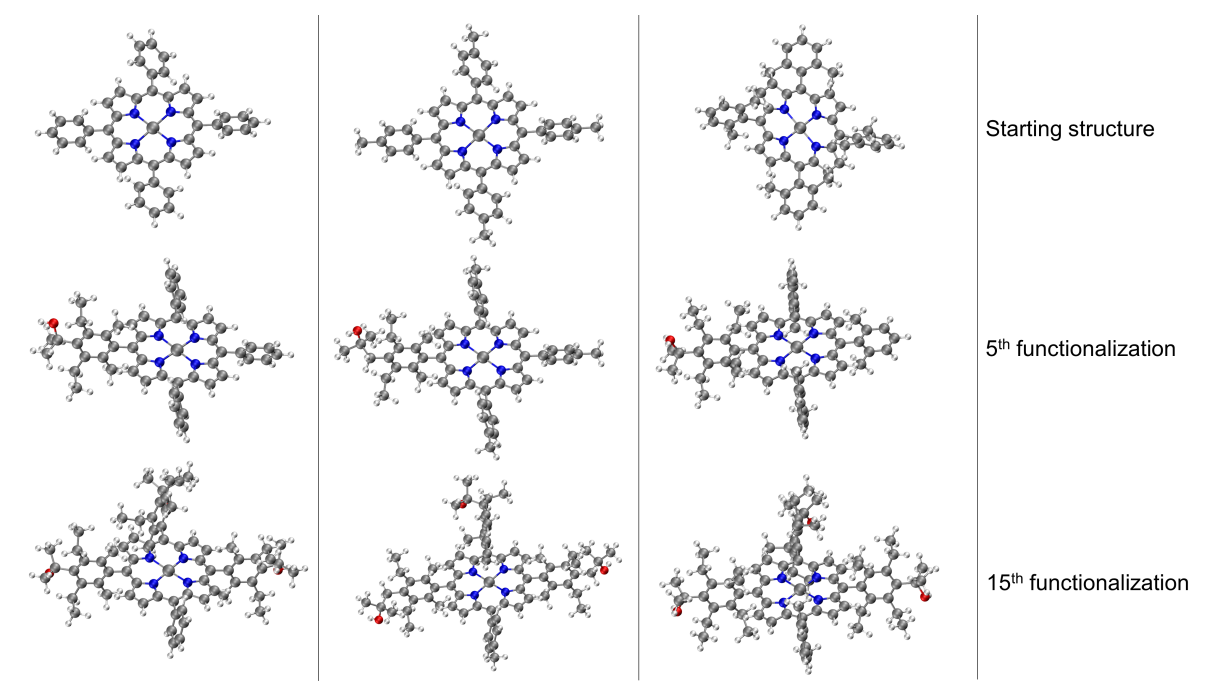

Figure 10: Functionalization strategy for Co porphyrin shown for 3 different skeletons. For each skeleton the 5 th functionalization and 15th functionalization are shown in 1 column. The phenyl rings are functionalized symmetrically. In the 5th functionalization the most left phenyl ring of the skeleton is functionalized, in the 10th functionalization the same substituents are placed on the upper phenyl ring and in the 15th functionalization on the most right phenyl ring. Color code used for elements: gray $=\mathrm{C}$ (metal center $=\mathrm{Co})$, white $=\mathrm{H}$, red $=\mathrm{O}$, dark-blue $=\mathrm{N}$ and turquoise $=\mathrm{F}$.

\subsubsection{Error propagation of serial functionalization}

The 1120 geometries were optimized using GFN2-xTB(THF). hRMSD between FF and GFN2XTB optimzied geometries was computed to compare the quality of the FF geometries generated by ChemSpaX, giving $\mu=1.28$, and $\sigma^{2}=0.54$. Upon detailed analysis of the hRMSD it was observed that the hRMSD increases nearly linear for each subsequent functionalization on a skeleton. The error introduced by placing a new substituent group is thus propagated upon the next placement of a substituent. An example is shown in Figure 11 where the hRMSD for each skeleton is plotted. The first 10 blocks of plots show the increasing hRMSD for each functionalization on a given skeleton. The last block shows the hRMSDs for all 10 skeletons, showing how the error increases almost linearly upon each functionalization regardless of the skeleton used for functionaliziation..

This finding could help users in devising an optimal strategy to get more accurate geometries at a balanced computational cost when using ChemSpaX. One can determine when an extra geometry optimization with a higher-level method, such as GFN2-xTB, is needed in between functionalizations by setting an hRMSD threshold. When this threshold is reached, the higherlevel optimization method can be used to reduce the hRMSD and the functionalization can be continued with the optimized intermediate as a new skeleton. 

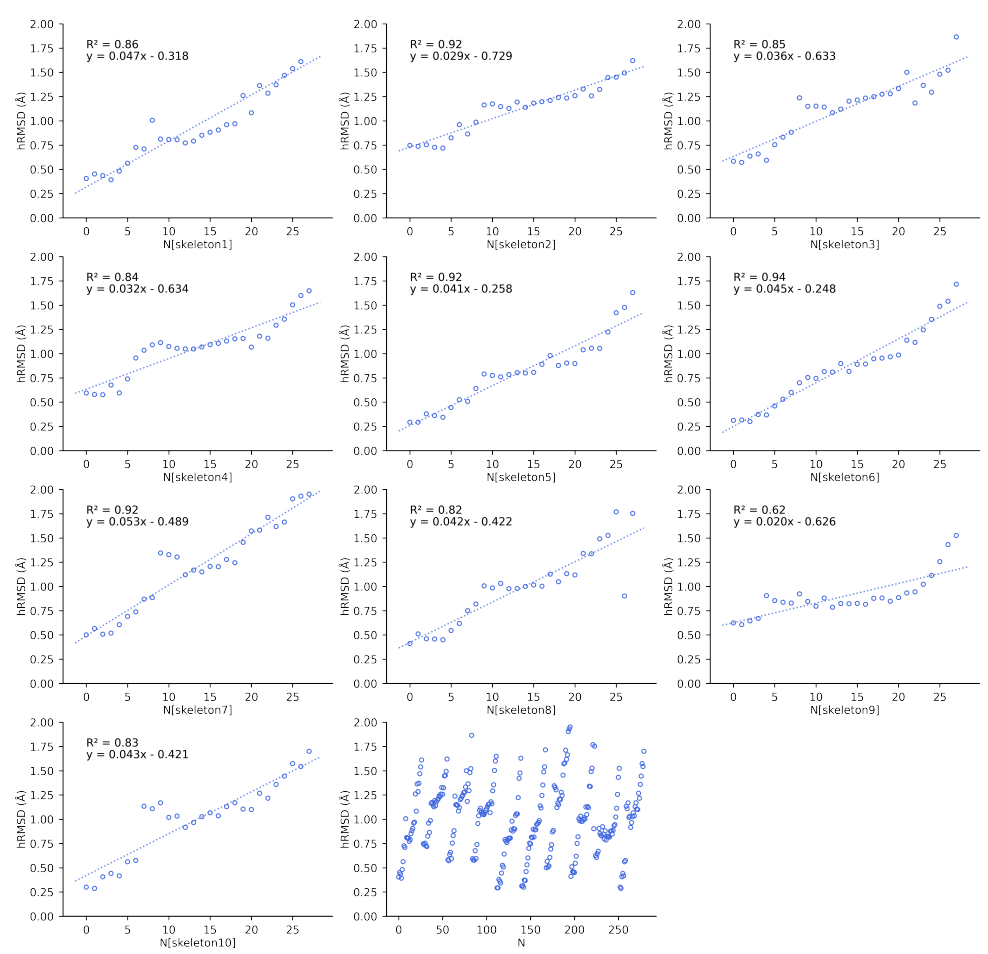

Figure 11: Increasing hRMSD for each functionalization on a given skeleton. Where $\mathrm{N}$ is the number of functionalizations, starting from 0.10 skeletons were created and 28 functionalizations were done for each skeleton. The first 10 blocks each represent a skeleton, while the last block on the bottom shows the increasing hRMSD for each skeleton grouped in 1 figure. After every 28th functionalization ( $0 \leq \mathrm{N} \leq 279$ ), a new skeleton is functionalized.

Analyzing hRMSD with the number of atoms in a structure we were able to identify 3 groups of data, namely:

1. hRMSD $<1.5 \AA$ (low)

2. $1.5 \AA<$ hRMSD $<2.0 \AA$ (middle)

3. hRMSD $>2.0 \AA$ (high)

A plot of hRMSD versus the number of atoms is shown in Figure 12. For smaller complexes ( $<150$ atoms) the hRMSD grows linearly with the number of atoms. For larger complexes (> 150 atoms) hRMSDs can range from low to high with the highest hRMSDs being found in the heaviest functionalized structures with a higher number of atoms. The error introduced by ChemSpaX upon functionalization is thus propagated, but the hRMSD remains below $3.34 \AA$, even after 28 functionalizations on the same skeleton.

Structure overlay plots of the FF geometries (silver) and the GFN2-xTB optimized geometries 


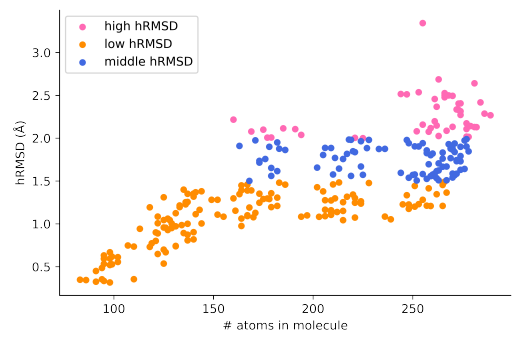

Figure 12: Plot of hRMSD against the number of atoms in a complex. The three hRMSD data groups are color coded accordingly.

(green) are shown in Figure 13. The upper half of the figure shows structure overlay plots of complexes with $<200$ atoms while the lower half shows complexes with $>200$ atoms, and diverse hRMSDs.
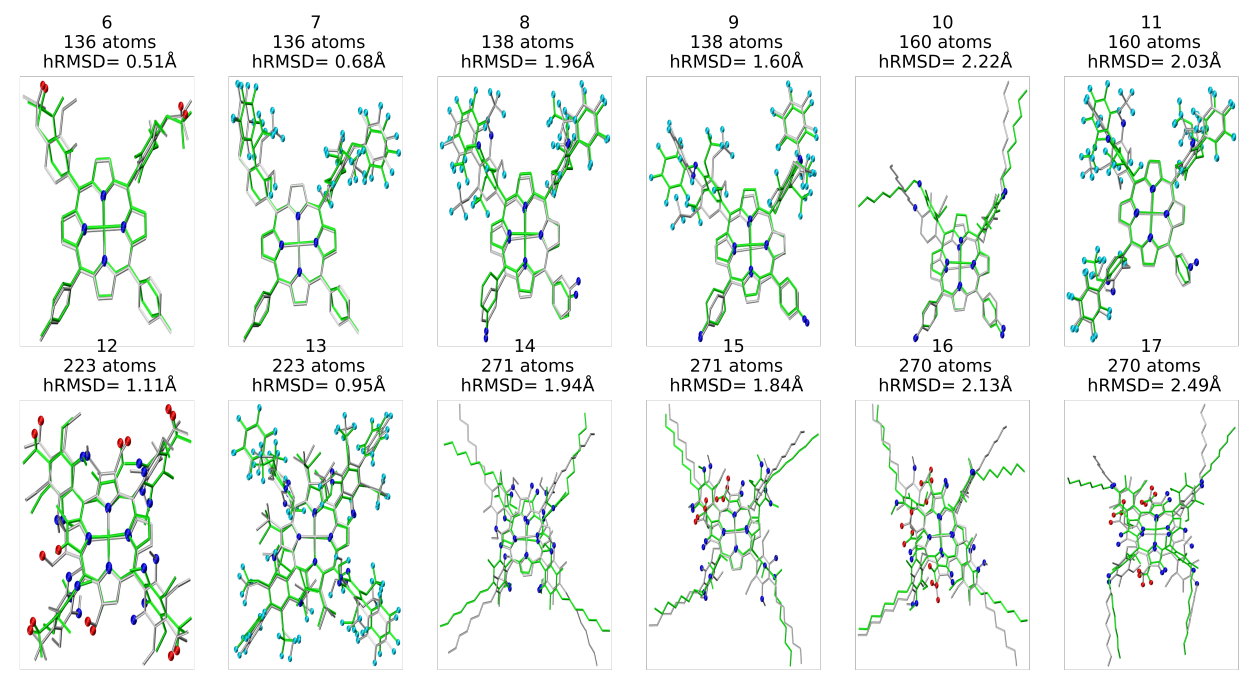

Figure 13: Structure overlay plots of selected Co porphyrin complexes. ChemSpaX generated (FF) structures (silver) are plotted against GFN2-xTB optimized (silver) structures. The upper half of the figure consists of structures with $<200$ atoms and the lower half of the figure shows structures $>200$ atoms. Color code used for elements: red $=\mathrm{O}$, dark-blue $=\mathrm{N}$ and turquoise $=\mathrm{F}$.

\subsubsection{HOMO-LUMO gap prediction}

Functional inorganic materials that are used for photocatalysis or photovoltaics require knowledge of the HOMO-LUMO gap, since this gap determines which wavelength of light is absorbed $[89,90,91]$. Additionally, the HOMO-LUMO gap can be used to predict the stability of TM complexes [92]. HOMO-LUMO gap prediction based on only the molecular structure as input using statistical methods, which avoids computationally expensive quantum chemistry calculations, can thus be a great resource to screen and develop functional inorganic materials [93].

We performed DFT based SP calculations on GFN2-xTB optimized geometries of 280 por- 
phyrin complexes out of the 1120 complexes generated using ChemSpaX. It was found that the hRMSD (error between ChemSpaX generated FF structures and GFN2-xTB optimized structures) for 280 selected structures has a moderate correlation with the DFT computed HOMOLUMO gap $(\mathrm{R}=-0.46)$ on the GFN2-xTB optimzied geometry. This indicates that this hRMSD could potentially be a feature in advanced machine learning applications for electronic property prediction. Additional analysis for prediction of the HOMO-LUMO gap can be found in the SI.

\subsection{M2L4 cage}

The versatility of ChemSpaX is shown by the automated placement of substituents without introducing steric hindrance on a geometry that is more complex. An M2L4 cage was functionalized at 16 sites with various substituent groups. The results are shown in figure Figure 14. This serial functionalization yielded 16 structures and these were further optimized using GFN-FF and GFN2-xTB(GAS). The hRMSDs between geometries generated by workflow of ChemSpaX (FF) were calculated against the GFN-FF and GFN2-XTB optimization methods. The statistics shown for each optimization method in Table 1 reveal that the ChemSpaX generated FF geometries are closer to the GFN2-xTB optimized geometries compared to GFN-FF optimized geometries.
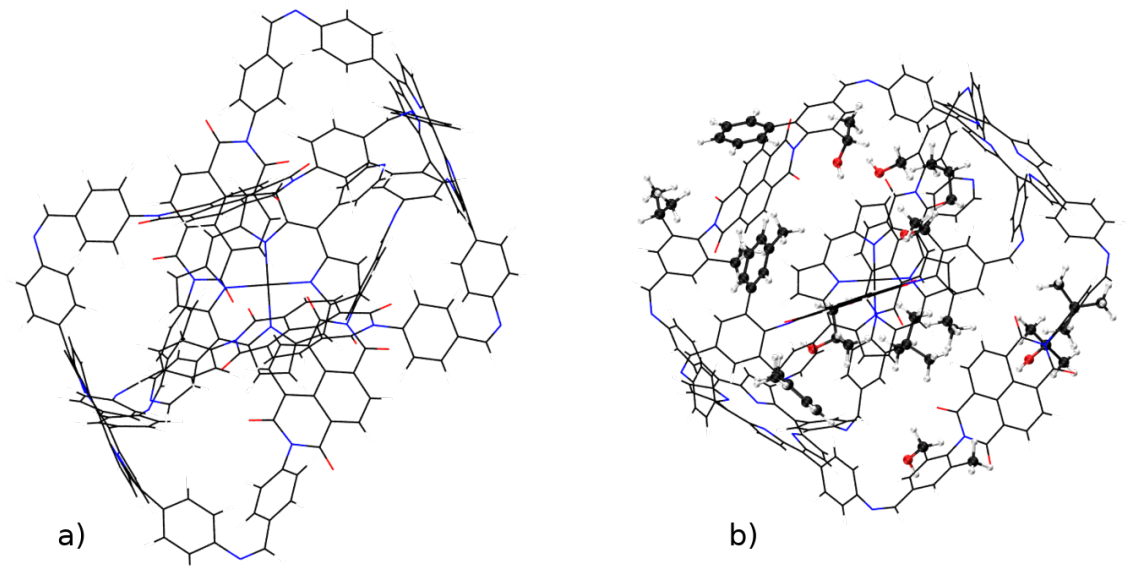

Figure 14: A visualization of the functionalized M2L4 cage which shows a) the input skeleton and b) the GFN2-xTB optimized geometry after placement of 16 substituents. The newly placed substituents are shown in a distinguished representation.

Table 1: Statistics for the hRMSD between various methods. The two optimization methods that are compared to each other are shown in the first row.

\begin{tabular}{c|c|c|c}
\hline & GFN-FF v GFN2-xTB & FF v GFN2-xTB & FF v GFN-FF \\
\hline$\mu$ & $2.54 \AA$ & $2.14 \AA$ & $0.83 \AA$ \\
$\sigma^{2}$ & $0.34 \AA$ & $0.25 \AA$ & $0.26 \AA$ \\
Max. hRMSD & $3.18 \AA$ & $2.46 \AA$ & $1.37 \AA$ \\
\hline
\end{tabular}




\section{Summary and conclusions}

In this research, an automated Python-based workflow for the exploration of local chemical space is presented. ChemSpaX can place substituents on specific sites of many structures based on initial user input and uses FF optimization to optimize newly placed substituents. Use cases were shown by using a data augmented approach which utilized fast GFN2-xTB optimizations to compare structures generated by ChemSpaX. For selected use cases a comparison was also done against DFT optimized structures. Descriptors such as the (free) energy of reaction that can be used for high-throughput screening were studied in more detail for some of the presented use cases.

For the pincer complexes the quality of geometries generated by ChemSpaX was assessed using $\Delta \Delta \mathrm{E}$ of reaction and hRMSDs. The $\Delta \Delta \mathrm{E}$ and hRMSDs indicated a good agreement between ChemSpaX generated FF structures and GFN2-xTB optimized structures for RuPNP. For the Mn-pincers it was observed that hRMSDs were in agreement with GFN2-xTB, although the $\Delta \Delta \mathrm{E}$ of reactions throw caution on direct use of ChemSpaX generated geometries in studying reaction energetics. A strong linear scaling of $\Delta \mathrm{E}$ and $\Delta \mathrm{G}\left(\mathrm{R}^{2}=0.99\right.$ for both RuPNP and the Mn-pincers) was found. This correlation was found for both DFT and GFN2-xTB calculations. Due to this correlation, $\Delta \mathrm{G}$ can be replaced by $\Delta \mathrm{E}$ in high-throughput screening, which would lower the consumption of computational resources significantly.

Functionalized Co porphyrins generated by ChemSpaX were compared against GFN2-xTB optimized structures. The errors introduced upon placement of substituents (hRMSD) were investigated for these Co porphyrins. This investigation showed a nearly linear increase in hRMSD for serial functionalizations done on the same skeleton. Using this analysis, a user can devise the optimal strategy to employ a higher-level optimization method based on a hRMSD threshold, to balance speed and accuracy. hRMSD was found to be a potential feature in advanced machine learning applications to predict HOMO-LUMO gap. Additionally, it has been presented that structures generated by ChemSpaX in this research are reasonably close to GFN2-xTB optimized structures and that diverse molecualr scaffolds can be functionalized using ChemSpaX.

To conclude, ChemSpaX can be used to generate satisfactory 3D geometric representations in the local chemical space of a given molecular scaffold, particularly including TM complexes. The generated structures, in conjunction with quantum chemical and statistical methods, can be used to generate structure-property databases enabling data-driven chemical design and discovery. There is room for improvement in ChemSpaX, which is in the early stages of development. Currently, when the user wants to functionalize multiple skeletons, ChemSpaX waits for the previous skeleton to be fully functionalized before moving on to the next. This poses an opportunity for parallelization of the code which is work in progress. Additionally, we are working on implementing a more flexible approach where the user can choose the frequency of intermediate optimizations with a high-level method based on a predicted hRMSD threshold.

With the development of ChemSpaX, an important challenge in HTS related to the generation of reasonably accurate 3D molecular geometries is addressed. This workflow is aimed at accelerating chemical space exploration and we hope that ChemSpaX will bolster the efforts of the catalysis and molecular modelling communities towards data-driven material discovery.

\section{Data and software availability}

The ChemSpaX workflow will be made public on our Github organization page: EPiCs-group (https://github.com/EPiCs-group). In addition to this manuscript, supporting information and all used datasets can be found: 
- full_datasets.zip contains datasets per investigated complex in Excel workbook format.

- geometry_files.zip contains geometry files for all structures in MDL Molfiles or XYZ format.

\section{Author contributions}

The code for ChemSpaX was written by A. V. K. and V. S. DFT \& xTB calculations were performed by A. V. K and V. S. Generation of functionalized structures, the compilation of datasets and analysis of DFT \& $x$ TB calculations was performed by A. V. K. under supervision of V. S. V. S. and E. A. P. conceived the project. E. A. P. played an advisory role and directed the project. All the authors discussed the results and wrote the manuscript.

\section{Conflicts of interest}

There are no conflicts of interest to declare.

\section{Acknowledgments}

V. S. acknowledges the ARC-CBBC project 2016.008 for funding. E.A.P. acknowledges the financial support from the European Research Council (ERC) under the European Union's Horizon 2020 Research and Innovation Programme (grant agreement no. 725686). This work was sponsored by NWO Domain Science for the use of the national computer facilities. The authors thank PRACE for DECl access in the DECl-16 call. The authors thank the PetaChem team for giving access to the TeraChem software package.

\section{References}

[1] Carl Poree and Franziska Schoenebeck. A Holy Grail in Chemistry: Computational Catalyst Design: Feasible or Fiction? Accounts of Chemical Research, 50(3):605-608, mar 2017. ISSN 0001-4842. doi: 10.1021/acs.accounts.6b00606. URL https://doi.org/10.1021/ acs . accounts. 6 b00606.

[2] John H Van Drie. Computer-aided drug design: the next 20 years. Journal of ComputerAided Molecular Design, 21(10):591-601, 2007. ISSN 1573-4951. doi: 10.1007/s10822007-9142-y. URL https://doi.org/10.1007/s10822-007-9142-y.

[3] Gregory Sliwoski, Sandeepkumar Kothiwale, Jens Meiler, and Edward W Lowe Jr. Computational methods in drug discovery. Pharmacological reviews, 66(1):334-395, dec 2013. ISSN 1521-0081. doi: 10.1124/pr.112.007336. URL https://pubmed.ncbi.nlm.nih. gov/24381236https://www.ncbi.nlm.nih.gov/pmc/articles/PMC3880464/.

[4] David E Clark. What has computer-aided molecular design ever done for drug discovery? Expert Opinion on Drug Discovery, 1(2):103-110, jul 2006. ISSN 1746-0441. doi: 10. 1517/17460441.1.2.103. URL https://doi.org/10.1517/17460441.1.2.103.

[5] David E Clark. What has virtual screening ever done for drug discovery? Expert Opinion on Drug Discovery, 3(8):841-851, aug 2008. ISSN 1746-0441. doi: 10.1517/17460441.3.8.841. URL https://doi.org/10.1517/17460441.3.8.841. 
[6] Anubhav Jain, Yongwoo Shin, and Kristin A Persson. Computational predictions of energy materials using density functional theory. Nature Reviews Materials, 1(1):15004, 2016. ISSN 2058-8437. doi: 10.1038/natrevmats.2015.4. URL https://doi.org/10.1038/ natrevmats.2015.4.

[7] Lei Cheng, Rajeev S Assary, Xiaohui Qu, Anubhav Jain, Shyue Ping Ong, Nav Nidhi Rajput, Kristin Persson, and Larry A Curtiss. Accelerating Electrolyte Discovery for Energy Storage with High-Throughput Screening. The Journal of Physical Chemistry Letters, 6(2):283-291, jan 2015. doi: 10.1021/jz502319n. URL https://doi.org/10.1021/jz502319n.

[8] Hossein Beidaghy Dizaji and Hannaneh Hosseini. A review of material screening in pure and mixed-metal oxide thermochemical energy storage (TCES) systems for concentrated solar power (CSP) applications. Renewable and Sustainable Energy Reviews, 98:9-26, 2018. ISSN 1364-0321. doi: https://doi.org/10.1016/j.rser.2018.09.004. URL http: //www.sciencedirect.com/science/article/pii/S136403211830652X.

[9] Johannes Hachmann, Roberto Olivares-Amaya, Sule Atahan-Evrenk, Carlos AmadorBedolla, Roel S Sánchez-Carrera, Aryeh Gold-Parker, Leslie Vogt, Anna M Brockway, and Alán Aspuru-Guzik. The Harvard Clean Energy Project: Large-Scale Computational Screening and Design of Organic Photovoltaics on the World Community Grid. The Journal of Physical Chemistry Letters, 2(17):2241-2251, sep 2011. doi: 10.1021/jz200866s. URL https://doi.org/10.1021/jz200866s.

[10] Kirstin Alberi, Marco Buongiorno Nardelli, Andriy Zakutayev, Lubos Mitas, Stefano Curtarolo, Anubhav Jain, Marco Fornari, Nicola Marzari, Ichiro Takeuchi, and Martin L Green. The 2019 materials by design roadmap. Journal of Physics D: Applied Physics, 52(1): 13001, 2018. ISSN 0022-3727.

[11] Peter Kirkpatrick and Clare Ellis. Chemical space. Nature, 432(7019):823, 2004. ISSN 1476-4687. doi: 10.1038/432823a. URL https://doi.org/10.1038/432823a.

[12] Christopher Zhou, William Grumbles, and Thomas Cundari. Using Machine Learning to Predict the $\mathrm{pKa}$ of $\mathrm{C}-\mathrm{H}$ Bonds. Relevance to Catalytic Methane Functionalization, jul 2020. URL https://chemrxiv.org/articles/preprint/Using_Machine_ Learning_to_Predict_the_pKa_of_C_H_Bonds_Relevance_to_Catalytic_ Methane_Functionalization/12646772https://chemrxiv.org/ndownloader/ files/23820425.

[13] O Anatole von Lilienfeld, Klaus-Robert Müller, and Alexandre Tkatchenko. Exploring chemical compound space with quantum-based machine learning. Nature Reviews Chemistry, 4(7):347-358, 2020. ISSN 2397-3358. doi: 10.1038/s41570-020-0189-9. URL https://doi.org/10.1038/s41570-020-0189-9.

[14] Gisbert Schneider. Automating drug discovery. Nature Reviews Drug Discovery, 17(2): 97-113, 2018. ISSN 1474-1784. doi: 10.1038/nrd.2017.232. URL https://doi.org/10. $1038 / \mathrm{nrd} .2017 .232$.

[15] Rafael Gómez-Bombarelli, Jennifer N Wei, David Duvenaud, José Miguel HernándezLobato, Benjamín Sánchez-Lengeling, Dennis Sheberla, Jorge Aguilera-Iparraguirre, Timothy D Hirzel, Ryan P Adams, and Alán Aspuru-Guzik. Automatic Chemical Design Using a Data-Driven Continuous Representation of Molecules. ACS Central Science, 
4(2):268-276, feb 2018. ISSN 2374-7943. doi: 10.1021/acscentsci.7b00572. URL https://doi.org/10.1021/acscentsci.7b00572.

[16] Thomas Blaschke, Marcus Olivecrona, Ola Engkvist, Jürgen Bajorath, and Hongming Chen. Application of Generative Autoencoder in De Novo Molecular Design. Molecular Informatics, 37(1-2):1700123, jan 2018. ISSN 1868-1743. doi: https://doi.org/10.1002/minf. 201700123. URL https://doi.org/10.1002/minf.201700123.

[17] Benjamin Meyer, Boodsarin Sawatlon, Stefan Heinen, O Anatole von Lilienfeld, and Clémence Corminboeuf. Machine learning meets volcano plots: computational discovery of cross-coupling catalysts. Chemical Science, 9(35):7069-7077, 2018. ISSN 2041-6520. doi: 10.1039/C8SC01949E. URL http://dx.doi.org/10.1039/C8SC01949E.

[18] Tobias Fink and Jean-Louis Reymond. Virtual Exploration of the Chemical Universe up to 11 Atoms of C, N, O, F: Assembly of 26.4 Million Structures (110.9 Million Stereoisomers) and Analysis for New Ring Systems, Stereochemistry, Physicochemical Properties, Compound Classes, and Drug Discovery. Journal of Chemical Information and Modeling, 47(2):342353, mar 2007. ISSN 1549-9596. doi: 10.1021/ci600423u. URL https://doi.org/10. 1021/ci600423u.

[19] Lorenz C Blum and Jean-Louis Reymond. 970 Million Druglike Small Molecules for Virtual Screening in the Chemical Universe Database GDB-13. Journal of the American Chemical Society, 131(25):8732-8733, jul 2009. ISSN 0002-7863. doi: 10.1021/ja902302h. URL https://doi.org/10.1021/ja902302h.

[20] Josep Arús-Pous, Thomas Blaschke, Silas Ulander, Jean-Louis Reymond, Hongming Chen, and Ola Engkvist. Exploring the GDB-13 chemical space using deep generative models. Journal of Cheminformatics, 11(1):20, 2019. ISSN 1758-2946. doi: 10.1186/s13321-0190341-z. URL https://doi.org/10.1186/s13321-019-0341-z.

[21] Pascal Friederich, Gabriel dos Passos Gomes, Riccardo De Bin, Alán Aspuru-Guzik, and David Balcells. Machine learning dihydrogen activation in the chemical space surrounding Vaska's complex. Chemical Science, 11(18):4584-4601, 2020. ISSN 2041-6520. doi: 10.1039/D0SC00445F. URL http://dx.doi.org/10.1039/D0SC00445F.

[22] Adrian Jinich, Benjamin Sanchez-Lengeling, Haniu Ren, Rebecca Harman, and Alán Aspuru-Guzik. A Mixed Quantum Chemistry/Machine Learning Approach for the Fast and Accurate Prediction of Biochemical Redox Potentials and Its Large-Scale Application to 315000 Redox Reactions. ACS Central Science, 5(7):1199-1210, jul 2019. ISSN 23747943. doi: 10.1021/acscentsci.9b00297. URL https://doi.org/10.1021/acscentsci. $9 \mathrm{~b} 00297$.

[23] Seoin Back, Kevin Tran, and Zachary W Ulissi. Toward a Design of Active Oxygen Evolution Catalysts: Insights from Automated Density Functional Theory Calculations and Machine Learning. ACS Catalysis, 9(9):7651-7659, sep 2019. doi: 10.1021/acscatal.9b02416. URL https://doi.org/10.1021/acscatal.9b02416.

[24] Craig A James, Richard Apodaca, Noel M O'Boyle, Andrew Dalke, John H Van Drie, Peter Ertl, Geoffrey R Hutchison, Greg Landrum, Chris Morley, Egon Willighagen, Hans De winter, Tim Vandermeersch, and John May. OpenSMILES specification, 2016. URL http://opensmiles.org/opensmiles.html. 
[25] Jan Jensen. xyz2mol: Convert Cartesian coordinates to one or more molecular graphs, 2020. URL https://github.com/jensengroup/xyz2mol.

[26] Efthymios I loannidis, Terry Z H Gani, and Heather J Kulik. molSimplify: A toolkit for automating discovery in inorganic chemistry. Journal of Computational Chemistry, 37(22): 2106-2117, 2016. ISSN 1096-987X. doi: 10.1002/jcc.24437. URL http://dx.doi.org/ $10.1002 / \mathrm{jcc} .24437$.

[27] Jon Paul Janet, Fang Liu, Aditya Nandy, Chenru Duan, Tzuhsiung Yang, Sean Lin, and Heather J Kulik. Designing in the Face of Uncertainty: Exploiting Electronic Structure and Machine Learning Models for Discovery in Inorganic Chemistry. Inorganic Chemistry, 58(16):10592-10606, aug 2019. ISSN 0020-1669. doi: 10.1021/acs.inorgchem.9b00109. URL https://doi.org/10.1021/acs.inorgchem.9b00109.

[28] Aditya Nandy, Chenru Duan, Jon Paul Janet, Stefan Gugler, and Heather J Kulik. Strategies and Software for Machine Learning Accelerated Discovery in Transition Metal Chemistry. Industrial \& Engineering Chemistry Research, 57(42):13973-13986, oct 2018. ISSN 0888-5885. doi: 10.1021/acs.iecr.8b04015. URL https://doi.org/10.1021/acs.iecr. $8 \mathrm{~b} 04015$.

[29] Jon Paul Janet, Terry Z H Gani, Adam H Steeves, Efthymios I loannidis, and Heather J Kulik. Leveraging Cheminformatics Strategies for Inorganic Discovery: Application to Redox Potential Design. Industrial \& Engineering Chemistry Research, 56(17):4898-4910, may 2017. ISSN 0888-5885. doi: 10.1021/acs.iecr.7b00808. URL https://doi.org/10. 1021/acs.iecr.7b00808.

[30] Jon Paul Janet, Qing Zhao, Efthymios I loannidis, and Heather J Kulik. Density functional theory for modelling large molecular adsorbate-surface interactions: a mini-review and worked example. Molecular Simulation, 43(5-6):327-345, apr 2017. ISSN 0892-7022. doi: 10.1080/08927022.2016.1258465. URL https://doi.org/10.1080/08927022.2016. 1258465 .

[31] Chelsea A Huff and Melanie S Sanford. Catalytic CO2 Hydrogenation to Formate by a Ruthenium Pincer Complex. ACS Catalysis, 3(10):2412-2416, oct 2013. doi: 10.1021/ cs400609u. URL https://doi.org/10.1021/cs400609u.

[32] Georgy A Filonenko, Robbert van Putten, Erik N Schulpen, Emiel J M Hensen, and Evgeny A Pidko. Highly Efficient Reversible Hydrogenation of Carbon Dioxide to Formates Using a Ruthenium PNP-Pincer Catalyst. ChemCatChem, 6(6):1485, jun 2014. ISSN 18673880. doi: https://doi.org/10.1002/cctc.201402265. URL https://doi.org/10.1002/ cctc. 201402265.

[33] Jacob Neumann, Christoph Bornschein, Haijun Jiao, Kathrin Junge, and Matthias Beller. Hydrogenation of Aliphatic and Aromatic Nitriles Using a Defined Ruthenium PNP Pincer Catalyst. European Journal of Organic Chemistry, 2015(27):5944-5948, sep 2015. ISSN 1434-193X. doi: https://doi.org/10.1002/ejoc.201501007. URL https://doi.org/10. 1002/ejoc. 201501007.

[34] Elisabetta Alberico, Alastair J J Lennox, Lydia K Vogt, Haijun Jiao, Wolfgang Baumann, Hans-Joachim Drexler, Martin Nielsen, Anke Spannenberg, Marek P Checinski, Henrik Junge, and Matthias Beller. Unravelling the Mechanism of Basic Aqueous Methanol Dehydrogenation Catalyzed by Ru-PNP Pincer Complexes. Journal of the American Chemical 
Society, 138(45):14890-14904, nov 2016. ISSN 0002-7863. doi: 10.1021/jacs.6b05692. URL https://doi.org/10.1021/jacs.6b05692.

[35] Zhihong Wei, Adiran de Aguirre, Kathrin Junge, Matthias Beller, and Haijun Jiao. Exploring the mechanisms of aqueous methanol dehydrogenation catalyzed by defined PNP Mn and Re pincer complexes under base-free as well as strong base conditions. Catalysis Science \& Technology, 8(14):3649-3665, 2018. ISSN 2044-4753. doi: 10.1039/C8CY00746B. URL http://dx.doi.org/10.1039/C8CY00746B.

[36] Anastasiya Agapova, Elisabetta Alberico, Anja Kammer, Henrik Junge, and Matthias Beller. Catalytic Dehydrogenation of Formic Acid with Ruthenium-PNP-Pincer Complexes: Comparing N-Methylated and NH-Ligands. ChemCatChem, 11(7):1910-1914, apr 2019. ISSN 1867-3880. doi: https://doi.org/10.1002/cctc.201801897. URL https: //doi.org/10.1002/cctc. 201801897.

[37] Annika Krieger, Vivek Sinha, Adarsh Kalikadien, and Evgeny Alexandrovich Pidko. Metalligand cooperative activation of $\mathrm{HX}(\mathrm{X}=\mathrm{H}, \mathrm{Br}, \mathrm{OR})$ bond on $\mathrm{Mn}$ based pincer complexes. Zeitschrift für anorganische und allgemeine Chemie, $\mathrm{n} / \mathrm{a}(\mathrm{n} / \mathrm{a})$, may 2021. ISSN 0044-2313. doi: https://doi.org/10.1002/zaac.202100078. URL https://doi.org/10.1002/zaac. 202100078.

[38] Vivek Sinha, Nitish Govindarajan, Bas de Bruin, and Evert Jan Meijer. How Solvent Affects $\mathrm{C}-\mathrm{H}$ Activation and Hydrogen Production Pathways in Homogeneous Ru-Catalyzed Methanol Dehydrogenation Reactions. ACS Catalysis, 8(8):6908-6913, aug 2018. doi: 10.1021/acscatal.8b01177. URL https://doi.org/10.1021/acscatal.8b01177.

[39] Nitish Govindarajan, Vivek Sinha, Monica Trincado, Hansjörg Grützmacher, Evert Jan Meijer, and Bas de Bruin. An In-Depth Mechanistic Study of Ru-Catalysed Aqueous Methanol Dehydrogenation and Prospects for Future Catalyst Design. ChemCatChem, 12(9):26102621, may 2020. ISSN 1867-3880. doi: https://doi.org/10.1002/cctc.202000057. URL https://doi.org/10.1002/cctc. 202000057.

[40] Georgy A Filonenko, Matthew P Conley, Christophe Copéret, Martin Lutz, Emiel J M Hensen, and Evgeny A Pidko. The impact of Metal-Ligand Cooperation in Hydrogenation of Carbon Dioxide Catalyzed by Ruthenium PNP Pincer. ACS Catalysis, 3(11):2522-2526, nov 2013. doi: 10.1021/cs4006869. URL https://doi.org/10.1021/cs4006869.

[41] Georgy A Filonenko, Emiel J M Hensen, and Evgeny A Pidko. Mechanism of CO2 hydrogenation to formates by homogeneous Ru-PNP pincer catalyst: from a theoretical description to performance optimization. Catalysis Science \& Technology, 4(10):3474-3485, 2014. ISSN 2044-4753. doi: 10.1039/C4CY00568F. URL http://dx.doi.org/10.1039/ C4CY00568F.

[42] Werner Bonrath, Jonathan Medlock, Jan Schütz, Bettina Wüstenberg, and Thomas Netscher. Hydrogenation in the Vitamins and Fine Chemicals Industry - An Overview. In Hydrogenation, pages 69-90. IntechOpen, oct 2012. ISBN 978-953-51-0785-9. doi: $10.5772 / 48751$.

[43] Felix Roessler. Catalysis in the Industrial Production of Pharmaceuticals and Fine Chemicals. CHIMIA International Journal for Chemistry, 50(3), 1996. URL https://www. ingentaconnect. com/content/scs/chimia/1996/00000050/00000003/art00012. 
[44] Reinaldo Machado, Kevin Heier, and Robert Broekhuis. Developments in Hydrogenation Technology for Fine-chemical and Pharmaceutical Applications. Current opinion in drug discovery \& development, 4:745-755, dec 2001.

[45] Albert Boddien, Christopher Federsel, Peter Sponholz, Dörthe Mellmann, Ralf Jackstell, Henrik Junge, Gabor Laurenczy, and Matthias Beller. Towards the development of a hydrogen battery. Energy \& Environmental Science, 5(10):8907-8911, 2012. ISSN 1754-5692. doi: 10.1039/C2EE22043A. URL http://dx.doi.org/10.1039/C2EE22043A.

[46] Björn Loges, Albert Boddien, Felix Gärtner, Henrik Junge, and Matthias Beller. Catalytic Generation of Hydrogen from Formic acid and its Derivatives: Useful Hydrogen Storage Materials. Topics in Catalysis, 53(13):902-914, 2010. ISSN 1572-9028. doi: 10.1007/ s11244-010-9522-8. URL https://doi.org/10.1007/s11244-010-9522-8.

[47] Ferenc Joó. Breakthroughs in Hydrogen Storage-Formic Acid as a Sustainable Storage Material for Hydrogen. ChemSusChem, 1(10):805-808, oct 2008. ISSN 1864-5631. doi: 10.1002/cssc.200800133. URL https://doi.org/10.1002/cssc. 200800133.

[48] Marcel Garbe, Kathrin Junge, and Matthias Beller. Homogeneous Catalysis by ManganeseBased Pincer Complexes. European Journal of Organic Chemistry, 2017(30):4344-4362, aug 2017. ISSN 1434-193X. doi: https://doi.org/10.1002/ejoc.201700376. URL https: //doi.org/10.1002/ejoc. 201700376.

[49] Monalisa Goswami, Christophe Rebreyend, and Bas de Bruin. Porphyrin Cobalt(III) "Nitrene Radical" Reactivity; Hydrogen Atom Transfer from Ortho-YH Substituents to the Nitrene Moiety of Cobalt-Bound Aryl Nitrene Intermediates ( $\mathrm{Y}=\mathrm{O}, \mathrm{NH}$ ). Molecules, 21(2):242, feb 2016. ISSN 1420-3049. doi: 10.3390/molecules21020242. URL http://dx.doi.org/ $10.3390 / \mathrm{molecules} 21020242$.

[50] Michael P Doyle and David C Forbes. Recent Advances in Asymmetric Catalytic Metal Carbene Transformations. Chemical Reviews, 98(2):911-936, apr 1998. ISSN 0009-2665. doi: 10.1021/cr940066a. URL https://doi.org/10.1021/cr940066a.

[51] Simone Fantauzzi, Alessandro Caselli, and Emma Gallo. Nitrene transfer reactions mediated by metallo-porphyrin complexes. Dalton Transactions, (28):5434-5443, 2009. ISSN 14779226. doi: 10.1039/B902929J. URL http://dx.doi.org/10.1039/B902929J.

[52] Matthias Otte, Petrus F Kuijpers, Oliver Troeppner, Ivana Ivanović-Burmazović, Joost N H Reek, and Bas de Bruin. Encapsulated Cobalt-Porphyrin as a Catalyst for Size-Selective Radical-type Cyclopropanation Reactions. Chemistry - A European Journal, 20(17):48804884, apr 2014. ISSN 0947-6539. doi: https://doi.org/10.1002/chem.201400055. URL https://doi.org/10.1002/chem.201400055.

[53] Valentinos Mouarrawis, Raoul Plessius, Jarl Ivar van der Vlugt, and Joost N H Reek. Confinement Effects in Catalysis Using Well-Defined Materials and Cages , 2018. URL https://www.frontiersin.org/article/10.3389/fchem.2018.00623.

[54] Matthias Otte, Petrus F Kuijpers, Oliver Troeppner, Ivana Ivanović-Burmazović, Joost $\mathrm{N} \mathrm{H}$ Reek, and Bas de Bruin. Encapsulation of Metalloporphyrins in a Self-Assembled Cubic M8L6 Cage: A New Molecular Flask for Cobalt-Porphyrin-Catalysed Radical-Type Reactions. Chemistry - A European Journal, 19(31):10170-10178, jul 2013. ISSN 09476539. doi: https://doi.org/10.1002/chem.201301411. URL https://doi.org/10.1002/ chem. 201301411. 
[55] Noel M O'Boyle, Michael Banck, Craig A James, Chris Morley, Tim Vandermeersch, and Geoffrey R Hutchison. The Open Babel Package, version 2.4.1, 2016. URL https:// openbabel.org/.

[56] Noel M O'Boyle, Michael Banck, Craig A James, Chris Morley, Tim Vandermeersch, and Geoffrey R Hutchison. Open Babel: An open chemical toolbox. Journal of Cheminformatics, 3(1):33, 2011. ISSN 1758-2946. doi: 10.1186/1758-2946-3-33. URL https://doi.org/ $10.1186 / 1758-2946-3-33$.

[57] A K Rappe, C J Casewit, K S Colwell, W A Goddard, and W M Skiff. UFF, a full periodic table force field for molecular mechanics and molecular dynamics simulations. Journal of the American Chemical Society, 114(25):10024-10035, dec 1992. ISSN 0002-7863. doi: 10.1021/ja00051a040. URL https://doi.org/10.1021/ja00051a040.

[58] Junmei Wang, Romain M Wolf, James W Caldwell, Peter A Kollman, and David A Case. Development and testing of a general amber force field. Journal of Computational Chemistry, 25(9):1157-1174, jul 2004. ISSN 0192-8651. doi: https://doi.org/10.1002/jcc.20035. URL https://doi.org/10.1002/jcc.20035.

[59] Christoph Bannwarth, Eike Caldeweyher, Sebastian Ehlert, Andreas Hansen, Philipp Pracht, Jakob Seibert, Sebastian Spicher, and Stefan Grimme. Extended tight-binding quantum chemistry methods. WIREs Computational Molecular Science, n/a(n/a):e01493, aug 2020. ISSN 1759-0876. doi: 10.1002/wcms.1493. URL https://doi.org/10.1002/wcms . 1493.

[60] Philipp Pracht, Eike Caldeweyher, Sebastian Ehlert, and Stefan Grimme. A Robust Non-Self-Consistent Tight-Binding Quantum Chemistry Method for large Molecules. ChemRxiv, jun 2019. doi: 10.26434/chemrxiv.8326202.v1. URL https: //chemrxiv.org/articles/preprint/A_Robust_Non-Self-Consistent_TightBinding_Quantum_Chemistry_Method_for_large_Molecules/8326202https: //chemrxiv.org/ndownloader/files/15605534.

[61] Stefan Grimme, Christoph Bannwarth, and Philip Shushkov. A Robust and Accurate TightBinding Quantum Chemical Method for Structures, Vibrational Frequencies, and Noncovalent Interactions of Large Molecular Systems Parametrized for All spd-Block Elements ( $Z=1-86)$. Journal of Chemical Theory and Computation, 13(5):1989-2009, may 2017. ISSN 1549-9618. doi: 10.1021/acs.jctc.7b00118. URL https://doi.org/10.1021/acs. jctc. 7b00118.

[62] Christoph Bannwarth, Sebastian Ehlert, and Stefan Grimme. GFN2-xTB-An Accurate and Broadly Parametrized Self-Consistent Tight-Binding Quantum Chemical Method with Multipole Electrostatics and Density-Dependent Dispersion Contributions. Journal of Chemical Theory and Computation, 15(3):1652-1671, mar 2019. ISSN 1549-9618. doi: 10.1021/acs.jctc.8b01176. URL https://doi.org/10.1021/acs.jctc.8b01176.

[63] Sebastian Spicher and Stefan Grimme. Robust Atomistic Modeling of Materials, Organometallic, and Biochemical Systems. Angewandte Chemie International Edition, 59(36):15665-15673, sep 2020. ISSN 1433-7851. doi: https://doi.org/10.1002/anie. 202004239. URL https://doi.org/10.1002/anie.202004239.

[64] W Clark Still, Anna Tempczyk, Ronald C Hawley, and Thomas Hendrickson. Semianalytical treatment of solvation for molecular mechanics and dynamics. Journal of 
the American Chemical Society, 112(16):6127-6129, aug 1990. ISSN 0002-7863. doi: 10.1021/ja00172a038. URL https://doi.org/10.1021/ja00172a038.

[65] T Ooi, M Oobatake, G Némethy, and H A Scheraga. Accessible surface areas as a measure of the thermodynamic parameters of hydration of peptides. Proceedings of the National Academy of Sciences, 84(10):3086 LP - 3090, may 1987. doi: 10.1073/pnas.84.10.3086. URL http://www. pnas.org/content/84/10/3086. abstract.

[66] M J Frisch, G W Trucks, H B Schlegel, G E Scuseria, M A Robb, J R Cheeseman, G Scalmani, V Barone, G A Petersson, H Nakatsuji, X Li, M Caricato, A V Marenich, J Bloino, B G Janesko, R Gomperts, B Mennucci, H P Hratchian, J V Ortiz, A F Izmaylov, J L Sonnenberg, D Williams-Young, F Ding, F Lipparini, F Egidi, J Goings, B Peng, A Petrone, T Henderson, D Ranasinghe, V G Zakrzewski, J Gao, N Rega, G Zheng, W Liang, M Hada, M Ehara, K Toyota, R Fukuda, J Hasegawa, M Ishida, T Nakajima, Y Honda, O Kitao, H Nakai, T Vreven, K Throssell, J A Montgomery Jr., J E Peralta, F Ogliaro, M J Bearpark, J J Heyd, E N Brothers, K N Kudin, V N Staroverov, T A Keith, R Kobayashi, J Normand, K Raghavachari, A P Rendell, J C Burant, S S Iyengar, J Tomasi, M Cossi, J M Millam, M Klene, C Adamo, R Cammi, J W Ochterski, R L Martin, K Morokuma, O Farkas, J B Foresman, and D J Fox. Gaussian 16 Revision C.01, 2016.

[67] A D Becke. Density-functional exchange-energy approximation with correct asymptotic behavior. Physical Review A, 38(6):3098-3100, sep 1988. doi: 10.1103/PhysRevA.38. 3098. URL https://link.aps.org/doi/10.1103/PhysRevA.38.3098.

[68] Florian Weigend and Reinhart Ahlrichs. Balanced basis sets of split valence, triple zeta valence and quadruple zeta valence quality for $\mathrm{H}$ to $\mathrm{Rn}$ : Design and assessment of accuracy. Physical Chemistry Chemical Physics, 7(18):3297-3305, 2005. ISSN 1463-9076. doi: 10. 1039/B508541A. URL http://dx.doi.org/10.1039/B508541A.

[69] Kasper P Jensen, Björn O Roos, and Ulf Ryde. Performance of density functionals for first row transition metal systems. The Journal of Chemical Physics, 126(1):14103, jan 2007. ISSN 0021-9606. doi: 10.1063/1.2406071. URL https://doi.org/10.1063/1.2406071.

[70] Michael Bühl and Hendrik Kabrede. Geometries of Transition-Metal Complexes from Density-Functional Theory. Journal of Chemical Theory and Computation, 2(5):12821290, sep 2006. ISSN 1549-9618. doi: 10.1021/ct6001187. URL https://doi.org/10. $1021 /$ ct6001187.

[71] Aleksandr V Marenich, Christopher J Cramer, and Donald G Truhlar. Universal Solvation Model Based on Solute Electron Density and on a Continuum Model of the Solvent Defined by the Bulk Dielectric Constant and Atomic Surface Tensions. The Journal of Physical Chemistry B, 113(18):6378-6396, may 2009. ISSN 1520-6106. doi: 10.1021/jp810292n. URL https://doi.org/10.1021/jp810292n.

[72] Burkhard Miehlich, Andreas Savin, Hermann Stoll, and Heinzwerner Preuss. Results obtained with the correlation energy density functionals of becke and Lee, Yang and Parr. Chemical Physics Letters, 157(3):200-206, 1989. ISSN 0009-2614. doi: https://doi. org/10.1016/0009-2614(89)87234-3. URL http://www.sciencedirect.com/science/ article/pii/0009261489872343.

[73] Eike Caldeweyher, Sebastian Ehlert, Andreas Hansen, Hagen Neugebauer, Sebastian Spicher, Christoph Bannwarth, and Stefan Grimme. A generally applicable atomic-charge 
dependent London dispersion correction. The Journal of Chemical Physics, 150(15):154122, apr 2019. ISSN 0021-9606. doi: 10.1063/1.5090222. URL https://doi.org/10.1063/ 1.5090222.

[74] Ivan S Ufimtsev and Todd J Martínez. Quantum Chemistry on Graphical Processing Units. 1. Strategies for Two-Electron Integral Evaluation. Journal of Chemical Theory and Computation, 4(2):222-231, feb 2008. ISSN 1549-9618. doi: 10.1021/ct700268q. URL https://doi.org/10.1021/ct700268q.

[75] Ivan S Ufimtsev and Todd J Martinez. Quantum Chemistry on Graphical Processing Units. 2. Direct Self-Consistent-Field Implementation. Journal of Chemical Theory and Computation, 5(4):1004-1015, apr 2009. ISSN 1549-9618. doi: 10.1021/ct800526s. URL https://doi.org/10.1021/ct800526s.

[76] Ivan S Ufimtsev and Todd J Martinez. Quantum Chemistry on Graphical Processing Units. 3. Analytical Energy Gradients, Geometry Optimization, and First Principles Molecular Dynamics. Journal of Chemical Theory and Computation, 5(10):2619-2628, oct 2009. ISSN 1549-9618. doi: 10.1021/ct9003004. URL https://doi.org/10.1021/ct9003004.

[77] Stefan Grimme, Jens Antony, Stephan Ehrlich, and Helge Krieg. A consistent and accurate ab initio parametrization of density functional dispersion correction (DFT-D) for the 94 elements H-Pu. The Journal of Chemical Physics, 132(15):154104, apr 2010. ISSN 00219606. doi: 10.1063/1.3382344. URL https://doi.org/10.1063/1.3382344.

[78] P Jeffrey Hay and Willard R Wadt. Ab initio effective core potentials for molecular calculations. Potentials for the transition metal atoms Sc to $\mathrm{Hg}$. The Journal of Chemical Physics, 82(1):270-283, jan 1985. ISSN 0021-9606. doi: 10.1063/1.448799. URL https://doi.org/10.1063/1.448799.

[79] Jimmy Charnley Kromann. Calculate Root-mean-square deviation (RMSD) of Two Molecules Using Rotation, GitHub, v1.3.2, 2020. URL https://github.com/charnley/ rmsd/releases/tag/rmsd-1.3.2.

[80] W Kabsch. A solution for the best rotation to relate two sets of vectors. Acta Crystallographica Section A, 32(5):922-923, sep 1976. ISSN 0567-7394. URL https: //doi.org/10.1107/S0567739476001873.

[81] Michael W Walker, Lejun Shao, and Richard A Volz. Estimating 3-D location parameters using dual number quaternions. CVGIP: Image Understanding, 54(3):358-367, 1991. ISSN 1049-9660. doi: https://doi.org/10.1016/1049-9660(91)90036-O. URL http://www . sciencedirect.com/science/article/pii/1049966091900360.

[82] Gabriel dos Passos Gomes, Robert Pollice, and Alán Aspuru-Guzik. Navigating through the Maze of Homogeneous Catalyst Design with Machine Learning. Trends in Chemistry, 3 (2):96-110, 2021. ISSN 2589-5974. doi: https://doi.org/10.1016/j.trechm.2020.12.006. URL https://www.sciencedirect.com/science/article/pii/S2589597420303166.

[83] Brian K Shoichet. Virtual screening of chemical libraries. Nature, 432(7019):862-865, 2004. ISSN 1476-4687. doi: 10.1038/nature03197. URL https://doi.org/10.1038/ nature03197.

[84] Vivek Sinha, Jochem Jan Laan, and Evgeny Pidko. Accurate and Rapid Prediction of pKa of Transition Metal Complexes: Semiempirical Quantum Chem- 
istry with a DataAugmented Approach, oct 2020. URL https://chemrxiv.org/ articles/preprint/Accurate_and_Rapid_Prediction_of_pKa_of_Transition_ Metal_Complexes_Semiempirical_Quantum_Chemistry_with_a_DataAugmented_ Approach/13087463https://chemrxiv.org/ndownloader/files/25048262.

[85] Satoru Hiroto, Yoshihiro Miyake, and Hiroshi Shinokubo. Synthesis and Functionalization of Porphyrins through Organometallic Methodologies. Chemical Reviews, 117(4):29103043, feb 2017. ISSN 0009-2665. doi: 10.1021/acs.chemrev.6b00427. URL https: //doi.org/10.1021/acs.chemrev.6b00427.

[86] David Dolphin. The Porphyrins V7: Biochemistry, Part B. Elsevier, 2012. ISBN 0323145612.

[87] Karl Kadish, Kevin M Smith, and Roger Guilard. The Porphyrin Handbook, Volume 3, volume 3. Elsevier, 2000. ISBN 0123932033.

[88] Robert Grubbs. Handbook of Metathesis Volume 1: Catalyst Development and Mechanism, aug 2003. URL https://doi.org/10.1002/9783527619481.

[89] Xijun Wang, Guozhen Zhang, Li Yang, Edward Sharman, and Jun Jiang. Material descriptors for photocatalyst/catalyst design. WIREs Computational Molecular Science, 8 (5):e1369, sep 2018. ISSN 1759-0876. doi: https://doi.org/10.1002/wcms.1369. URL https://doi.org/10.1002/wcms.1369.

[90] Albert Polman, Mark Knight, Erik C Garnett, Bruno Ehrler, and Wim C Sinke. Photovoltaic materials: Present efficiencies and future challenges. Science, 352(6283):aad4424, apr 2016. doi: 10.1126/science.aad4424. URL http://science.sciencemag.org/content/352/ 6283/aad4424. abstract.

[91] SeJin Ahn, Sunghun Jung, Jihye Gwak, Ara Cho, Keeshik Shin, Kyunghoon Yoon, Doyoung Park, Hyeonsik Cheong, and Jae Ho Yun. Determination of band gap energy (Eg) of Cu2ZnSnSe4 thin films: On the discrepancies of reported band gap values. Applied Physics Letters, 97(2):21905, jul 2010. ISSN 0003-6951. doi: 10.1063/1.3457172. URL https: //doi.org/10.1063/1.3457172.

[92] J S Griffith and L E Orgel. Ligand-field theory. Quarterly Reviews, Chemical Society, 11(4): 381-393, 1957. ISSN 0009-2681. doi: 10.1039/QR9571100381. URL http://dx.doi. org/10.1039/QR9571100381.

[93] Ya Zhuo, Aria Mansouri Tehrani, and Jakoah Brgoch. Predicting the Band Gaps of Inorganic Solids by Machine Learning. The Journal of Physical Chemistry Letters, 9(7):1668-1673, apr 2018. doi: 10.1021/acs.jpclett.8b00124. URL https://doi.org/10.1021/acs . jpclett.8b00124. 